EPJ manuscript No.

(will be inserted by the editor)

\title{
Partial decay widths of baryons in the spin-momentum operator expansion method
}

\author{
A.V. Anisovich ${ }^{1,2}$ and A.V. Sarantsev ${ }^{1,2}$ \\ 1 HISKP, Universität Bonn, D-53115 Bonn, \\ 2 Petersburg Nuclear Physics Institute, Gatchina, Russia
}

April 9, 2018

\begin{abstract}
The cross sections for photo- and pion-induced production of baryon resonances and their partial decay widths to the two-body and multi-body final states are calculated in the framework of the operator expansion method. The approach is fully relativistic invariant, and it allows us to perform combined analyses of different reactions imposing directly the analyticity and unitarity constraints. All formulae are given explicitly in the form used by the Crystal Barrel collaboration in the partial wave analysis.
\end{abstract}

\section{Introduction}

The detailed knowledge of the low-energy hadron masses and their decays is vital to construct and test QCD-inspired models for the nonperturbative regime. In recent years, appreciable progress has been achieved in meson spectroscopy where a new information was mostly obtained from the analysis of reactions with three or more particles in the final state. A prime example is the rich spectrum of new resonances obtained in the $\bar{p} p$ annihilation at rest [1,2,3,4,5,6,7] and in flight 8,9,10,11.

While in the meson sector the majority of states predicted by the quark models [12,13 has been observed as well as some extra states with respect to the $q \bar{q}$ classification, the situation in the baryon sector is dramatically different. Here a number of quark models predicts 14, 15, [16] much richer spectrum than that observed so far. In addition, certain models predict the existence of states which are - from the quark model point of view - exotic, like pentaquarks 17, heptaquarks 18, or nucleon-meson bound states [19. The lack of the predicted states can be an indication of new phenomena such as formation of diquark states [20,21,22] or specific interaction of colour particles at large distances 21 23 that lead to linear trajectories on $\left(n, M^{2}\right)$ (where $n$ is the number of a radial excitation) and $\left(J, M^{2}\right)$ planes. However, the explanation could be much simpler: the modern knowledge of the baryon spectrum is based mostly on the analysis of pion-induced reactions on the single meson production (mostly data obtained from the elastic pion-nucleon scattering) and it is quite possible that many states with weak coupling to the $\pi N$ channel escaped the detection.

Recently, a number of experiments on baryon spectroscopy has been initiated in several laboratories. A large

Correspondence to: andsar@hiskp.uni-bonn.de amount of new data is coming from photoproduction experiments such as CLAS, CB-ELSA, GRAAL, Mainz-

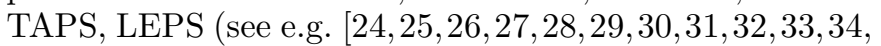
35 ). The analysis of the new photoproduction data and especially the data obtained from reactions with multiparticle final states is therefore one of the most urgent tasks in baryon spectroscopy.

The meson spectroscopy teaches us that the analysis of reactions with multiparticle final states cannot be done unambiguously without information about reactions with the two-body final states. The best way to impose such information is to perform a combined analysis of the set of reactions. This issue is even more important in baryon spectroscopy where often the polarization of initial or/and final particles is not detected. Here, even in the analysis of the two-body final states the combined analysis of the data from different channels plays a vital role. Thus, the development of a method which describes different reactions on the same basis is a key point in the search for new baryon states.

The method based on relativistic invariant operators which are constructed directly from the 4 -vectors of the particles was put forward in a set of articles (see 36 and reference therein). In the article [36, the operators for the photoproduction and pion-induced reactions were introduced and the amplitude angular dependences were calculated. The analysis of a large number of single meson photoproduction reactions performed in the framework of this method 37,38 reveals a number of new baryon states. Here, the resonances were parameterized as T-matrix poles with mass, width and the product of initial and final couplings as fitted parameters. However, such an observation should be confirmed by the combined analysis of the photoproduction and pion-induced reaction, with two and many particle final states. In this case, the couplings of the baryon resonances to different channels can be unambigu- 
ously defined, partial widths calculated and checked for consistency.

In this article, we develop the approach of 36 providing explicit expressions for the calculation of cross sections for different reactions and partial widths of the states. The obtained expressions can be directly used in the T- or Kmatrix combined analysis of the photo and pion induced reactions.

The article is organized as follows. In section 2, we provide general expressions for the calculation of cross sections and resonance widths in the framework of the operator approach. In section 3, we discuss resonance widths calculated with dispersion relations and give explicit expressions for the resonances decaying into spinless particles. The calculation of $\pi N$ partial widths of baryon states is given in Section 4 and $\gamma N$ partial widths in Section 5. The three-particle partial widths and correspondent singularities are discussed in Section 6.

\section{Cross section for transition amplitudes and the widths of resonances}

For the production of $m$ particles with the 4-momenta $q_{i}$ from two particles colliding with 4 -momenta $k_{1}$ and $k_{2}$, the cross section is given by

$$
d \sigma=\frac{(2 \pi)^{4}|A|^{2}}{4|\mathbf{k}| \sqrt{s}} d \Phi_{m}\left(P, q_{1} \ldots q_{m}\right), \quad P=k_{1}+k_{2},
$$

where $A$ is the transition amplitude, $\mathbf{k}$ is the 3 -momentum of the initial particle calculated in the centre-of-mass system (c.m.s.) of the reaction, and $s=P^{2}=\left(k_{1}+k_{2}\right)^{2}$. The cross section is calculated by averaging over polarizations of initial-state particles and summing over polarization of final-state particles. The invariant $\mathrm{m}$-particle phase space is given by

$$
d \Phi_{m}\left(P, q_{1} \ldots q_{m}\right)=\delta^{4}\left(P-\sum_{i=1}^{m} q_{i}\right) \prod_{i=1}^{m} \frac{d^{3} q_{i}}{(2 \pi)^{3} 2 q_{0 i}} .
$$

The amplitude for the transition from the initial state " in" to the final state "out" via a resonance with the total spin $J$, mass $M$ and width $\Gamma_{\text {tot }}$ has the form:

$$
A=\frac{g_{i n} Q_{\mu_{1} \ldots \mu_{n}}^{i n} F_{\nu_{1} \ldots \nu_{n}}^{\mu_{1} \ldots \mu_{n}} Q_{\nu_{1} \ldots \nu_{n}}^{o u t} g_{o u t}}{M^{2}-s-i M \Gamma_{\text {tot }}}
$$

Here $n=J$ for mesons, and $n=J-1 / 2$ for baryons, $g_{\text {in }}$ and $g_{\text {out }}$ are initial- and final-state couplings, $Q^{\text {in }}$ and $Q^{\text {out }}$ are operators which describe the production and decay processes and $F_{\nu_{1} \ldots \nu_{n}}^{\mu_{1} \ldots \mu_{n}}$ is the tensor part of the resonance propagator.

The standard formula for the decay of a resonance into $m$ particles is given by

$$
M \Gamma=\int \frac{(2 \pi)^{4}}{2}\left|A_{d e c}\right|^{2} d \Phi_{m}\left(P, q_{1} \ldots q_{m}\right)
$$

and, as in the case of the cross section, one has to sum over the polarizations of the final-state particles.
In the operator representation, the amplitude $A_{d e c}$ has the form

$$
A_{d e c}=\bar{\Psi}_{\mu_{1} \ldots \mu_{n}}^{(i)} Q_{\mu_{1} \ldots \mu_{n}} g
$$

where $\Psi_{\mu_{1} \ldots \mu_{n}}^{(i)}$ is polarization tensor of the resonance (conventionally, we call it the polarization wave function), $Q_{\mu_{1} \ldots \mu_{n}}$ is the operator of the transition of the resonance into final state, and $g$ is the corresponding coupling constant. For example, if $Q=Q^{\text {out }}$ and $g=g_{\text {out }}$, eq. (4) provides the partial width for the resonance decay into the final state and for $Q=Q^{i n}$ and $g=g_{i n}$ the partial widths for its decay into the initial state.

The tensor part of the propagator is determined by the polarization tensor as follows:

$$
F_{\nu_{1} \ldots \nu_{n}}^{\mu_{1} \ldots \mu_{n}}=\sum_{i=1}^{2 J+1} \Psi_{\mu_{1} \ldots \mu_{n}}^{(i)} \bar{\Psi}_{\nu_{1} \ldots \nu_{n}}^{(i)}
$$

where the summation is performed over all possible polarizations of the resonance. We use the following normalization for the polarization tensor:

$$
\bar{\Psi}_{\mu_{1} \ldots \mu_{n}}^{(i)} \Psi_{\mu_{1} \ldots \mu_{n}}^{(j)}=(-1)^{n} \delta_{i j}
$$

With this choice of the sign, the tensor part of the propagator differs from that introduced in 36. by a factor $(-1)^{n}$. However, the present definition is more convenient to calculate the unitarity condition, and we keep it in further calculations.

Multiplying the amplitude squared by $\Psi_{\alpha_{1} \ldots \alpha_{n}}^{(i)} \bar{\Psi}_{\beta_{1} \ldots \beta_{n}}^{(i)}$ and summing over polarizations we obtain:

$$
\begin{aligned}
& F_{\beta_{1} \ldots \beta_{n}}^{\alpha_{1} \ldots \alpha_{n}} M \Gamma=\int \frac{(2 \pi)^{4}}{2} d \Phi_{m}\left(P, q_{1} \ldots q_{m}\right) g^{2}(s) \times \\
& \sum_{i=1}^{2 J+1} \Psi_{\alpha_{1} \ldots \alpha_{n}}^{(i)} \bar{\Psi}_{\mu_{1} \ldots \mu_{n}}^{(i)} Q_{\mu_{1} \ldots \mu_{n}} \otimes Q_{\nu_{1} \ldots \nu_{n}} \Psi_{\nu_{1} \ldots \nu_{n}}^{(i)} \bar{\Psi}_{\beta_{1} \ldots \beta_{n}}^{(i)}
\end{aligned}
$$

Here, the expression $Q_{\mu_{1} \ldots \mu_{n}} \otimes Q_{\nu_{1} \ldots \nu_{n}}$ assumes summation over polarizations of the final particles. Due to orthogonality of the polarization tensors,

$$
\int \bar{\Psi}_{\mu_{1} \ldots \mu_{n}}^{(i)} Q_{\mu_{1} \ldots \mu_{n}} \otimes Q_{\nu_{1} \ldots \nu_{n}} \Psi_{\nu_{1} \ldots \nu_{n}}^{(j)} d \Phi_{m}\left(P, q_{1} \ldots q_{m}\right) \sim \delta_{i j}(9)
$$

the product of the polarization tensors can be substituted by

$$
\Psi_{\nu_{1} \ldots \nu_{n}}^{(i)} \bar{\Psi}_{\beta_{1} \ldots \beta_{n}}^{(i)} \rightarrow \sum_{j=1}^{2 J+1} \Psi_{\nu_{1} \ldots \nu_{n}}^{(j)} \bar{\Psi}_{\beta_{1} \ldots \beta_{n}}^{(j)}=F_{\beta_{1} \ldots \beta_{n}}^{\nu_{1} \ldots \nu_{n}}
$$

Finally, we obtain

$$
\begin{aligned}
F_{\beta_{1} \ldots \beta_{n}}^{\alpha_{1} \ldots \alpha_{n}} M \Gamma= & \int \frac{(2 \pi)^{4}}{2} d \Phi_{m}\left(P, q_{1} \ldots q_{m}\right) g^{2}(s) \times \\
& F_{\mu_{1} \ldots \mu_{n}}^{\alpha_{1} \ldots \alpha_{n}} Q_{\mu_{1} \ldots \mu_{n}} \otimes Q_{\nu_{1} \ldots \nu_{n}} F_{\beta_{1} \ldots \beta_{n}}^{\nu_{1} \ldots \nu_{n}} .
\end{aligned}
$$

This is the basic equation for the calculation of partial widths of resonances. Another form of this equation can 
be obtained after the convolution of both sides of equation (11) with the metric tensors $g_{\alpha_{1} \beta_{1}} \ldots g_{\alpha_{n} \beta_{n}}$ and taking into account the properties (6) and (7):

$$
\begin{aligned}
(2 J+1) M \Gamma= & \int \frac{(2 \pi)^{4}}{2} d \Phi_{m}\left(P, q_{1} \ldots q_{m}\right) g^{2}(s) \times \\
& Q_{\mu_{1} \ldots \mu_{n}} \otimes Q_{\nu_{1} \ldots \nu_{n}} F_{\mu_{1} \ldots \mu_{n}}^{\nu_{1} \ldots \nu_{n}} .
\end{aligned}
$$

Then the cross section for $2 \rightarrow m$ particle transition can be rewritten as

$$
\begin{aligned}
& \sigma=\int \frac{(2 \pi)^{4}}{4|\mathbf{k}| \sqrt{s}} d \Phi_{m}\left(P, q_{1} \ldots q_{m}\right) g_{\text {in }}^{2} Q_{\mu_{1} \ldots \mu_{n}}^{i n} \times \\
& \frac{F_{\nu_{1} \ldots \nu_{n}}^{\mu_{1} \ldots \mu_{n}} Q_{\nu_{1} \ldots \nu_{n}}^{\text {out }} \otimes Q_{\alpha_{1} \ldots \alpha_{n}}^{\text {out }} F_{\beta_{1} \ldots \beta_{n}}^{\alpha_{1} \ldots \alpha_{n}}}{\left(M^{2}-s\right)^{2}+\left(M \Gamma_{\text {tot }}\right)^{2}} Q_{\beta_{1} \ldots \beta_{n}}^{\text {in }} g_{\text {out }}^{2}= \\
& \frac{g_{\text {in }}^{2}}{2|\mathbf{k}| \sqrt{s}} \frac{Q_{\mu_{1} \ldots \mu_{n}}^{\text {in }} F_{\beta_{1} \ldots \beta_{n}}^{\mu_{1} \ldots \mu_{n}} M \Gamma_{\text {out }} Q_{\beta_{1} \ldots \beta_{n}}^{\text {in }}}{\left(M^{2}-s\right)^{2}+\left(M \Gamma_{\text {tot }}\right)^{2}}
\end{aligned}
$$

The two-body phase space of particles with masses $m_{1}$ and $m_{2}$ is equal to

$$
\frac{(2 \pi)^{4}}{2} d \Phi_{2}\left(P, k_{1}, k_{2}\right)=\rho\left(s, m_{1}, m_{2}\right) \frac{d \Omega}{4 \pi},
$$

where the invariant form of the $\rho$ function is given by

$$
\rho\left(s, m_{1}, m_{2}\right)=\frac{\sqrt{\left.\left(s-\left(m_{1}+m_{2}\right)^{2}\right)\right)\left(s-\left(m_{1}-m_{2}\right)^{2}\right)}}{16 \pi s} .
$$

This function can be also expressed through momentum of the particles in the c.m.s of the reaction:

$$
\rho\left(s, m_{1}, m_{2}\right)=\frac{1}{16 \pi} \frac{2|\mathbf{k}|}{\sqrt{s}} .
$$

Then, we can use eq.(12) to calculate partial width for decays into the initial-state particles. If they have spins $s_{1}$ and $s_{2}$, the cross section is calculated by averaging over their polarizations. After the summation over spin variables, one has for the partial width:

$$
\sigma=\frac{2 J+1}{\left(2 s_{1}+1\right)\left(2 s_{2}+1\right)} \frac{4 \pi}{|\mathbf{k}|^{2}} \frac{M^{2} \Gamma_{\text {in }} \Gamma_{\text {out }}}{\left(M^{2}-s\right)^{2}+\left(M \Gamma_{\text {tot }}\right)^{2}} .
$$

It is the standard equation for the contribution of a resonance with spin $J$ to the cross section.

\section{The width of Breit-Wigner resonance}

\subsection{Rescatering of particles and Breit-Wigner states}

The amplitude for the rescattering of two spinless particles with total momentum $P\left(s=P^{2}\right)$ via scalar resonance with the bare mass $M_{0}$ (Fig. 1) can be written as an infinite sum:

$$
A(s)=\frac{g^{2}(s)}{M_{0}^{2}-s}+\frac{g(s)}{M_{0}^{2}-s} B(s) \frac{g(s)}{M_{0}^{2}-s}+\ldots
$$

where the $B(s)$ function corresponds to the loop in the intermediate state. It can be calculated, for example, in terms of the Feynman integral. However, for our purpose it is convenient to use the dispersion relation technique where the real part of the amplitude can be constructed as a dispersion integral over the imaginary part. The imaginary part of the loop diagram is equal to

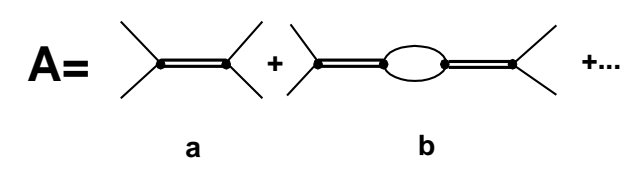

Fig. 1. Resonance rescattering diagrams

$$
\operatorname{ImB}(s)=g^{2}(s) \int \frac{(2 \pi)^{4}}{2} d \Phi_{2}\left(P, k_{1}, k_{2}\right)
$$

where $k_{i}$ and $m_{i}$ are the momenta and masses of the particles in the loop. Then, $B(s)$ can be written as the integral

$$
B(s)=\int_{\left(m_{1}+m_{2}\right)^{2}}^{\infty} \frac{d s^{\prime}}{\pi} \frac{\operatorname{Im} B\left(s^{\prime}\right)}{s^{\prime}-s-i 0}
$$

In the considered spinless particle case, the amplitude corresponding to (18) can be easily calculated

$$
A(s)=\frac{g^{2}(s)}{M_{0}^{2}-s} \frac{1}{1-\frac{B(s)}{M_{0}^{2}-s}}=\frac{g^{2}(s)}{M_{0}^{2}-s-B(s)} .
$$

The real part of the $B(s)$ function has left-hand singularities in the complex- $s$ plane and is a smooth function in the physical region. Therefore in the resonance region

$$
\operatorname{Re} B(s) \simeq \operatorname{Re} B\left(M^{2}\right)+\operatorname{Re} B^{\prime}\left(M^{2}\right)\left(s-M^{2}\right)
$$

and the amplitude can be written as

$$
A(s)=\frac{\tilde{g}^{2}(s)}{M^{2}-s-i M \Gamma},
$$

where

$$
\begin{aligned}
& M^{2}=M_{0}^{2}-\operatorname{Re} B\left(M^{2}\right), \\
& M \Gamma=\operatorname{ImB}\left(M^{2}\right) /\left(1+\operatorname{Re} B^{\prime}\left(M^{2}\right)\right), \\
& \tilde{g}^{2}(s)=g^{2}(s) /\left(1+\operatorname{Re} B^{\prime}\left(M^{2}\right)\right)
\end{aligned}
$$

The first line of eq.24 defines the position of the resonance, the imaginary part of $B(s)$ defines the width of the state. 
Now consider the general case. The amplitude which describes the scattering via a resonance with total spin $J$ is given by

$$
\begin{aligned}
& A(s)=g_{\text {in }} Q_{\mu_{1} \ldots \mu_{n}}^{\text {in }} \frac{F_{\nu_{1} \ldots \nu_{n}}^{\mu_{1} \ldots \mu_{n}}}{M_{0}^{2}-s} Q_{\nu_{1} \ldots \nu_{n}}^{\text {out }} g_{\text {out }}+ \\
& g_{\text {in }} Q_{\mu_{1} \ldots \mu_{n}}^{\text {in }} \frac{F_{\nu_{1} \ldots \nu_{n}}^{\mu_{1} \ldots \mu_{n}}}{M_{0}^{2}-s} \tilde{B}_{\xi_{1} \ldots \xi_{n}}^{\nu_{1} \ldots \nu_{n}} \frac{F_{\beta_{1} \ldots \beta_{n}}^{\xi_{1} \ldots \xi_{n}}}{M_{0}^{2}-s} Q_{\beta_{1} \ldots \beta_{n}}^{\text {out }} g_{\text {out }}+\ldots
\end{aligned}
$$

Here, $F_{\nu_{1} \ldots \nu_{n}}^{\mu_{1} \ldots \mu_{n}}$ is the tensor part of the propagator, $Q^{i n}$, $Q^{\text {out }}$ are vertex operators for the initial and final states ( $n=J$ for boson and $n=J-1 / 2$ for fermion resonances). We assume also that the vertex operators include the polarization tensors of the initial and final particles.

The imaginary part of the loop diagram for the intermediate state with $m$ particles is given by

$$
\begin{aligned}
\operatorname{Im} \tilde{B}_{\xi_{1} \ldots \xi_{n}}^{\nu_{1} \ldots \nu_{n}} & =\int \frac{(2 \pi)^{4}}{2} g^{2}(s) d \Phi_{m} Q_{\nu_{1} \ldots \nu_{n}} \otimes Q_{\xi_{1} \ldots \xi_{n}}, \\
d \Phi_{m} & \equiv d \Phi_{m}\left(P, k_{1}, \ldots, k_{m}\right) .
\end{aligned}
$$

Here, $g(s)$ and $Q$ are the coupling and vertex operator, respectively, for the decay of a resonance into the intermediate state. As before, the definition $Q_{\nu_{1} \ldots \nu_{n}} \otimes Q_{\xi_{1} \ldots \xi_{n}}$ assumes summation over polarizations of the intermediate particles. In the pure elastic case, the intermediate state operator is equal to $Q=Q^{i n}=Q^{\text {out }}$ but generally, the $B$-function is equal to the sum of loop diagrams over all possible decay modes.

Let us define the $B(s)$-function as follows:

$$
\begin{gathered}
F_{\beta_{1} \ldots \beta_{n}}^{\mu_{1} \ldots \mu_{n}} \operatorname{Im} B(s)=F_{\nu_{1} \ldots \nu_{n}}^{\mu_{1} \ldots \mu_{n}} \operatorname{Im} \tilde{B}_{\xi_{1} \ldots \xi_{n}}^{\nu_{1} \ldots \nu_{n}} F_{\beta_{1} \ldots \beta_{n}}^{\xi_{1} \ldots \xi_{n}}= \\
F_{\nu_{1} \ldots \nu_{n}}^{\mu_{1} \ldots \mu_{n}} \int \frac{(2 \pi)^{4}}{2} g^{2}(s) d \Phi_{m} Q_{\nu_{1} \ldots \nu_{n}} \otimes Q_{\xi_{1} \ldots \xi_{n}} F_{\beta_{1} \ldots \beta_{n}}^{\xi_{1} \ldots \xi_{n}}
\end{gathered}
$$

Using this equation, one can convolute all tensor factors into one structure, so one obtains:

$$
\begin{array}{r}
A(s)=g_{\text {in }} Q_{\mu_{1} \ldots \mu_{n}}^{i n} \frac{F_{\nu_{1} \ldots \nu_{n}}^{\mu_{1} \ldots \mu_{n}}}{M_{0}^{2}-s} Q_{\nu_{1} \ldots \nu_{n}}^{\text {out }} g_{\text {out }}(1+ \\
\left.\frac{B(s)}{M_{0}^{2}-s}+\frac{B(s)}{M_{0}^{2}-s} \frac{B(s)}{M_{0}^{2}-s}+\ldots\right)= \\
g_{\text {in }} Q_{\mu_{1} \ldots \mu_{n}}^{\text {in }} \frac{F_{\nu_{1} \ldots \nu_{n}}^{\mu_{1} \ldots \mu_{n}}}{M_{0}^{2}-s-B(s)} Q_{\nu_{1} \ldots \nu_{n}}^{\text {out }} g_{\text {out }} .
\end{array}
$$

As before, the imaginary part of the B-function defines the width of the state, and we obtain the standard BreitWigner expression.

\subsection{Decay of the resonance into two spinless particles}

For the rescattering of two spinless particles the vertex is defined by the orbital momentum operators only. These operators satisfy the symmetry, orthogonality and traceless properties (see 39] for more detail):

$$
\begin{aligned}
& X_{\mu_{1} \ldots \mu_{i} \ldots \mu_{j} \ldots \mu_{L}}^{(L)}=X_{\mu_{1} \ldots \mu_{j} \ldots \mu_{i} \ldots \mu_{L}}^{(L)} . \\
& P_{\mu_{i}} X_{\mu_{1} \ldots \mu_{i} \ldots \mu_{L}}^{(L)}=0 . \\
& g_{\mu_{i} \mu_{j}} X_{\mu_{1} \ldots \mu_{i} \ldots \mu_{j} \ldots \mu_{L}}^{(L)}=0
\end{aligned}
$$

where $L$ is the orbital momentum. The second property means that the operators $X$ are constructed from vectors and tensors orthogonal to the total momentum. In the case of the two-particle final state, only one vector of such a type can be constructed:

$$
k_{\mu}^{\perp}=\frac{1}{2} g_{\mu \nu}^{\perp}\left(k_{1}-k_{2}\right)_{\nu}, \quad g_{\mu \nu}^{\perp}=g_{\mu \nu}-\frac{P_{\mu} P_{\nu}}{s},
$$

where $k_{1}$ and $k_{2}$ are the momenta of the constituents.

The orbital angular momentum operators for $L \leq 3$ are:

$$
\begin{aligned}
X^{(0)} & =1, \quad X_{\mu}^{(1)}=k_{\mu}^{\perp} \\
X_{\mu_{1} \mu_{2}}^{(2)} & =\frac{3}{2}\left(k_{\mu_{1}}^{\perp} k_{\mu_{2}}^{\perp}-\frac{1}{3} k_{\perp}^{2} g_{\mu_{1} \mu_{2}}^{\perp}\right), \\
X_{\mu_{1} \mu_{2} \mu_{3}}^{(3)} & =\frac{5}{2}\left[k_{\mu_{1}}^{\perp} k_{\mu_{2}}^{\perp} k_{\mu_{3}}^{\perp}\right. \\
& \left.-\frac{k_{\perp}^{2}}{5}\left(g_{\mu_{1} \mu_{2}}^{\perp} k_{\mu_{3}}^{\perp}+g_{\mu_{1} \mu_{3}}^{\perp} k_{\mu_{2}}^{\perp}+g_{\mu_{2} \mu_{3}}^{\perp} k_{\mu_{1}}^{\perp}\right)\right] .
\end{aligned}
$$

The operators $X_{\mu_{1} \ldots \mu_{L}}^{(L)}$ for $L \geq 1$ can be written in the form of the recurrency expression:

$$
\begin{aligned}
X_{\mu_{1} \ldots \mu_{L}}^{(L)} & =k_{\alpha}^{\perp} Z_{\mu_{1} \ldots \mu_{L}}^{\alpha}, \\
Z_{\mu_{1} \ldots \mu_{L}}^{\alpha} & =\frac{2 L-1}{L^{2}}\left(\sum_{i=1}^{L} X_{\mu_{1} \ldots \mu_{i-1} \mu_{i+1} \ldots \mu_{L}}^{(L-1)} g_{\mu_{i} \alpha}^{\perp}-\right. \\
\frac{2}{2 L-1} \sum_{\substack{i, j=1 \\
i<j}}^{L} g_{\mu_{i} \mu_{j}}^{\perp} & \left.X_{\mu_{1} \ldots \mu_{i-1} \mu_{i+1} \ldots \mu_{j-1} \mu_{j+1} \ldots \mu_{L} \alpha}^{(L-1)}\right) .
\end{aligned}
$$

Other useful properties of the orbital momentum operators are listed in Appendix.

The projection operator $O_{\nu_{1} \ldots \nu_{L}}^{\mu_{1} \ldots \mu_{L}}$ is constructed from the metric tensors $g_{\mu \nu}^{\perp}$ and has the following properties:

$$
\begin{gathered}
X_{\mu_{1} \ldots \mu_{L}}^{(L)} O_{\nu_{1} \ldots \nu_{L}}^{\mu_{1} \ldots \mu_{L}}=X_{\nu_{1} \ldots \nu_{L}}^{(L)}, \\
O_{\alpha_{1} \ldots \alpha_{L}}^{\mu_{1} \ldots \mu_{L}} O_{\nu_{1} \ldots \nu_{L}}^{\alpha_{1} \ldots \alpha_{L}}=O_{\nu_{1} \ldots \nu_{L}}^{\mu_{1} \ldots \mu_{L}} .
\end{gathered}
$$

The projection operator projects any tensor with $n$ indices onto tensors which satisfy the properties (29). For the lowest states,

$$
\begin{aligned}
O & =1 \quad O_{\nu}^{\mu}=g_{\mu \nu}^{\perp} \\
O_{\nu_{1} \nu_{2}}^{\mu_{1} \mu_{2}} & =\frac{1}{2}\left(g_{\mu_{1} \nu_{1}}^{\perp} g_{\mu_{2} \nu_{2}}^{\perp}+g_{\mu_{1} \nu_{2}}^{\perp} g_{\mu_{2} \nu_{1}}^{\perp}-\frac{2}{3} g_{\mu_{1} \mu_{2}}^{\perp} g_{\nu_{1} \nu_{2}}^{\perp}\right)
\end{aligned}
$$

For higher states, the operator can be calculated using the recurrent expression:

$$
\begin{aligned}
& O_{\nu_{1} \ldots \nu_{L}}^{\mu_{1} \ldots \mu_{L}}=\frac{1}{L^{2}}\left(\sum_{i, j=1}^{L} g_{\mu_{i} \nu_{j}}^{\perp} O_{\nu_{1} \ldots \nu_{j-1} \nu_{j+1} \ldots \nu_{L}}^{\mu_{1} \ldots \mu_{i-1} \mu_{i+1} \ldots \mu_{L}}-\right. \\
& \frac{4}{(2 L-1)(2 L-3)} \times \\
& \left.\sum_{\substack{i<j \\
k<m}}^{L} g_{\mu_{i} \mu_{j}}^{\perp} g_{\nu_{k} \nu_{m}}^{\perp} O_{\nu_{1} \ldots \nu_{k-1} \nu_{k+1} \ldots \nu_{m-1} \nu_{m+1} \ldots \nu_{L}}^{\mu_{1} \ldots \mu_{i-1} \mu_{i+1} \ldots \mu_{j-1} \mu_{j+1} \ldots \mu_{L}}\right)
\end{aligned}
$$


The tensor part of the boson propagator is defined by the projection operator. Let us write it as

$$
F_{\nu_{1} \ldots \nu_{L}}^{\mu_{1} \ldots \mu_{L}}=(-1)^{L} O_{\nu_{1} \ldots \nu_{L}}^{\mu_{1} \ldots \mu_{L}}
$$

This definition differs from the expression given in 36 by the factor $(-1)^{L}$. This choice simplifies the expressions for amplitudes given in 36 where this factor was explicitly included in all final expressions. Furthermore, this definition guarantees that the width of the resonance (when it is calculated from the vertices) is a positive value.

The production of the two $X$-operators integrated over solid angle (which is equivalent to the integration over internal momenta) depends on external momenta and the metric tensor only. Therefore, it must be proportional to the projection operator. After straightforward calculations, we obtain:

$$
\int \frac{d \Omega}{4 \pi} X_{\mu_{1} \ldots \mu_{L}}^{(L)}\left(k^{\perp}\right) X_{\nu_{1} \ldots \nu_{L}}^{(L)}\left(k^{\perp}\right)=\frac{\alpha_{L} k_{\perp}^{2 L}}{2 L+1} O_{\nu_{1} \ldots \nu_{L}}^{\mu_{1} \ldots \mu_{L}},
$$

where

$$
\alpha_{L}=\prod_{l=1}^{L} \frac{2 l-1}{l}
$$

The width of the state is calculated by means of eq. (11) using the properties (33):

$$
M \Gamma=(-1)^{L} \frac{\alpha_{L} g^{2}(s)}{2 L+1} k_{\perp}^{2 L} \rho\left(s, m, m_{2}\right),
$$

where $\rho\left(s, m_{1}, m_{2}\right)$ is defined by eqs. 1415) and $g(s)$ is the coupling.

Let us introduce the positive value $|\mathbf{k}|^{2}$ :

$$
|\mathbf{k}|^{2}=-k_{\perp}^{2}=\frac{\left[s-\left(m_{1}+m_{2}\right)^{2}\right]\left[s-\left(m_{1}-m_{2}\right)^{2}\right]}{4 s} .
$$

In the c.m.s. of the reaction, $\mathbf{k}$ corresponds to the momentum of the particle. In other systems we use this definition only in the sense of $|\mathbf{k}| \equiv \sqrt{-k_{\perp}^{2}}$, and therefore it is relativistic invariant positive value. Then we obtain

$$
M \Gamma=\frac{\alpha_{L} g^{2}(s)}{2 L+1}|\mathbf{k}|^{2 L} \rho\left(s, m_{1}, m_{2}\right)
$$

as the width of a state. The amplitude for the rescattering of constituents with the initial relative momentum $k$ and final relative momentum $q$ is equal to

$$
\begin{aligned}
A(s) & =g(s) X_{\mu_{1} \ldots \mu_{L}}^{(L)}\left(k^{\perp}\right) \frac{(-1)^{L} O_{\nu_{1} \ldots \nu_{L}}^{\mu_{1} \ldots \mu_{l}}}{M^{2}-s-i M \Gamma} X_{\nu_{1} \ldots \nu_{L}}^{(L)}\left(q^{\perp}\right) g(s) \\
& =g(s) \frac{\alpha_{L} P_{L}(z)(|\mathbf{k}||\mathbf{q}|)^{L}}{M^{2}-s-i M \Gamma} g(s) .
\end{aligned}
$$

Here $P_{L}(z)$ are Legendre polynomials and $z$ is equal to

$$
z=\frac{k^{\perp} q^{\perp}}{\sqrt{k_{\perp}^{2}} \sqrt{q_{\perp}^{2}}} .
$$

In the c.m.s. of the resonance, $z$ is the cosine of the angle between the momenta of initial-state and final-state particles. To obtain eq. (42), we used the following property of the $X$-operators:

$$
X_{\mu_{1} \ldots \mu_{L}}^{(L)}\left(k^{\perp}\right) X_{\mu_{1} \ldots \mu_{L}}^{(L)}\left(q^{\perp}\right)=\alpha_{L}\left(\sqrt{k_{\perp}^{2}} \sqrt{q_{\perp}^{2}}\right)^{L} P_{L}(z) .
$$

The total cross section for the elastic scattering is equal to:

$$
\begin{aligned}
\sigma & =\frac{(2 \pi)^{4}}{4|\mathbf{k}| \sqrt{s}} \int \frac{g^{4}(s) \alpha_{L}^{2} P_{L}^{2}(z)(|\mathbf{k}||\mathbf{q}|)^{2 L}}{\left(M^{2}-s\right)^{2}+(M \Gamma)^{2}} d \Phi_{2}\left(P, q_{1}, q_{2}\right) \\
& =\frac{4 \pi}{|\mathbf{k}|^{2}}(2 L+1) \frac{(M \Gamma)^{2}}{\left(M^{2}-s\right)^{2}+(M \Gamma)^{2}}
\end{aligned}
$$

\section{Partial widths of the baryon resonances}

\subsection{The structure of the fermion propagator}

The structure of the fermion propagator $\mathcal{P}_{\nu_{1} \ldots \nu_{n}}^{\mu_{1} \ldots \mu_{n}}$ was considered in details in 36. The propagator is defined as

$$
\mathcal{P}_{\nu_{1} \ldots \nu_{n}}^{\mu_{1} \ldots \mu_{n}}=\frac{F_{\nu_{1} \ldots \nu_{n}}^{\mu_{1} \ldots \mu_{n}}}{M^{2}-s-i M \Gamma}
$$

where

$$
F_{\nu_{1} \ldots \nu_{n}}^{\mu_{1} \ldots \mu_{n}}=(-1)^{n} \frac{\sqrt{s}+\hat{P}}{2 \sqrt{s}} O_{\xi_{1} \ldots \xi_{n}}^{\mu_{1} \ldots \mu_{n}} T_{\beta_{1} \ldots \beta_{n}}^{\xi_{1} \ldots \xi_{n}} O_{\nu_{1} \ldots \nu_{n}}^{\beta_{1} \ldots \beta_{n}} .
$$

Here, $(\sqrt{s}+\hat{P})$ corresponds to the numerator of fermion propagator describing the particle with $J=1 / 2$ and $n=$ $J-1 / 2(\sqrt{s}=M$ for the stable particle). We define

$$
\begin{aligned}
T_{\beta_{1} \ldots \beta_{n}}^{\xi_{1} \ldots \xi_{n}} & =\frac{n+1}{2 n+1}\left(g_{\xi_{1} \beta_{1}}-\frac{n}{n+1} \sigma_{\xi_{1} \beta_{1}}\right) \prod_{i=2}^{n} g_{\xi_{i} \beta_{i}} \\
\sigma_{\alpha_{i} \alpha_{j}} & =\frac{1}{2}\left(\gamma_{\alpha_{i}} \gamma_{\alpha_{j}}-\gamma_{\alpha_{j}} \gamma_{\alpha_{i}}\right)
\end{aligned}
$$

As in 36, we introduced the factor $1 /(2 \sqrt{s})$ in the propagator which removes the divergency of this function at large energies. For the stable particle it means that bispinors are normalized as follows:

$$
\bar{u}\left(k_{N}\right) u\left(k_{N}\right)=1, \sum_{\text {polarizations }} u\left(k_{N}\right) \bar{u}\left(k_{N}\right)=\frac{m+\hat{k}_{N}}{2 m}
$$

Here and below, $\hat{k} \equiv \gamma_{\mu} k_{\mu}$.

It is useful to list the properties of the fermion propagator:

$$
\begin{aligned}
& P_{\mu_{i}} F_{\nu_{1} \ldots \nu_{n}}^{\mu_{1} \ldots \mu_{n}}=P_{\nu_{j}} F_{\nu_{1} \ldots \nu_{n}}^{\mu_{1} \ldots \mu_{n}}=0, \\
& \gamma_{\mu_{i}} F_{\nu_{1} \ldots \nu_{n}}^{\mu_{1} \ldots \mu_{n}}=F_{\nu_{1} \ldots \nu_{n}}^{\mu_{1} \ldots \mu_{n}} \gamma_{\nu_{j}}=0, \\
& F_{\alpha_{1} \ldots \alpha_{n}}^{\mu_{1} \ldots \mu_{n}} F_{\nu_{1} \ldots \nu_{n}}^{\alpha_{1} \ldots \alpha_{n}}=(-1)^{n} F_{\nu_{1} \ldots \nu_{n}}^{\mu_{1} \ldots \mu_{n}} \\
& \hat{P} F_{\nu_{1} \ldots \nu_{n}}^{\mu_{1} \ldots \mu_{n}}=\sqrt{s} F_{\nu_{1} \ldots \nu_{n}}^{\mu_{1} \ldots \mu_{n}} .
\end{aligned}
$$




\section{$4.2 \pi N$ partial widths of baryon resonances}

The operators which describe the decay of a baryon into the $\pi N$ system were introduced in [36]. The states with $J=L+1 / 2$, where $L$ is the orbital momentum of the $\pi N$ system, are called ' $+^{\prime}$ states $\left(1 / 2^{-}, 3 / 2^{+}, 5 / 2^{-}, \ldots\right)$. The states with $J=L-1 / 2$ are called '-' states $\left(1 / 2^{+}, 3 / 2^{-}\right.$, $\left.5 / 2^{+}, \ldots\right)$. The correspondent vertices are $(n=J-1 / 2)$ :

$$
\begin{aligned}
& N_{\mu_{1} \ldots \mu_{n}}^{+}\left(k^{\perp}\right) u\left(k_{N}\right)=X_{\mu_{1} \ldots \mu_{n}}^{(n)}\left(k^{\perp}\right) u\left(k_{N}\right) . \\
& N_{\mu_{1} \ldots \mu_{n}}^{-}\left(k^{\perp}\right) u\left(k_{N}\right)=i \gamma_{5} \gamma_{\nu} X_{\nu \mu_{1} \ldots \mu_{n}}^{(n+1)}\left(k^{\perp}\right) u\left(k_{N}\right) .
\end{aligned}
$$

Here, $u\left(k_{N}\right)$ is the bispinor of the final-state nucleon.

The width is defined by the equation (11) which for the case of $\pi N$ scattering has the form

$$
\begin{aligned}
& F_{\nu_{1} \ldots \nu_{n}}^{\mu_{1} \ldots \mu_{n}} M \Gamma_{\pi N}^{ \pm}=F_{\xi_{1} \ldots \xi_{n}}^{\mu_{1} \ldots \mu_{n}} \int \frac{d \Omega}{4 \pi} \tilde{N}_{\xi_{1} \ldots \xi_{n}}^{ \pm} \times \\
& \frac{\hat{k}_{N}+m_{N}}{2 m_{N}} N_{\beta_{1} \ldots \beta_{n}}^{ \pm} \rho\left(s, m_{\pi}, m_{N}\right) g^{2}(s) F_{\nu_{1} \ldots \nu_{n}}^{\beta_{1} \ldots \beta_{n}} .
\end{aligned}
$$

Here, we use $\tilde{N}$ to define the vertex which is different from $N$ by the order of gamma matrices.

The momentum of the nucleon can be decomposed in the total momentum $\mathrm{P}$ and momentum $k^{\perp}$ as:

$$
k_{N \mu}=\frac{k_{N 0}}{\sqrt{s}} P_{\mu}+k_{\mu}^{\perp}, \quad k_{N 0}=\frac{s+m_{N}^{2}-m_{\pi}^{2}}{2 \sqrt{s}}
$$

where

$$
k_{\mu}^{\perp}=\frac{1}{2}\left(k_{N}-k_{\pi}\right)_{\mu}-\frac{m_{N}^{2}-m_{\pi}^{2}}{2 s} P_{\mu} .
$$

For '+' states the calculation can be easily performed using eq. (37), the last property from eqs. (50) and the condition that integral over the odd number of $k_{\mu}^{\perp}$ vectors vanishes. Then,

$$
M \Gamma_{\pi N}^{+}=\frac{\alpha_{n}}{2 n+1}|\mathbf{k}|^{2 n} \frac{m_{N}+k_{N 0}}{2 m_{N}} \rho\left(s, m_{\pi}, m_{N}\right) g^{2}(s) .
$$

For the '-' states the calculations are more complicated. Using the formulae given in Appendix, one obtains finally

$$
M \Gamma_{\pi N}^{-}=\frac{\alpha_{n+1}}{n+1}|\mathbf{k}|^{2 n+2} \frac{m_{N}+k_{N 0}}{2 m_{N}} \rho\left(s, m_{\pi}, m_{N}\right) g^{2}(s) \text {. }
$$

The definition of the propagator in the form (47) provides a positive magnitude of the resonance widths and correct position of the poles of the scattering amplitude. Let us write with this definition the expression for the scattering amplitude. The partial amplitude for the $\pi N$ scattering from the initial state with relative momentum $k^{\perp}$ into the final state with momentum $q^{\perp}$ is defined by:

$$
\begin{aligned}
A_{\pi N} & =\sum_{n} A_{n}^{+} B W_{n}^{+}(s)+A_{n}^{-} B W_{n}^{-}(s), \\
A_{n}^{ \pm} & =\bar{u}\left(k_{1}\right) \tilde{N}_{\mu_{1} \ldots \mu_{n}}^{ \pm}\left(k^{\perp}\right) F_{\nu_{1} \ldots \nu_{n}}^{\mu_{1} \ldots \mu_{n}} N_{\nu_{1} \ldots \nu_{n}}^{ \pm}\left(q^{\perp}\right) u\left(q_{1}\right) .
\end{aligned}
$$

Here, $B W_{n}^{ \pm}(s)$ is the energy-dependent part of the amplitude.

In the c.m.s. of the reaction (see 36] for more detail), this amplitude can be rewritten as

$$
\begin{aligned}
& A_{\pi N}=\omega^{*}[G(s, t)+H(s, t) i(\sigma \mathbf{n})] \omega^{\prime}, \\
& G(s, t)=\sum_{L}\left[(L+1) F_{L}^{+}(s)-L F_{L}^{-}(s)\right] P_{L}(z), \\
& H(s, t)=\sum_{L}\left[F_{L}^{+}(s)+F_{L}^{-}(s)\right] P_{L}^{\prime}(z),
\end{aligned}
$$

where $\omega$ and $\omega^{\prime}$ are nonrelativistic spinors and $\mathbf{n}$ is a unit vector normal to the decay plane. The $F$-functions are defined as follows:

$$
\begin{aligned}
F_{L}^{+} & =(|\mathbf{k} \| \mathbf{q}|)^{L} \sqrt{\chi_{i} \chi_{f}} \frac{\alpha_{L}}{2 L+1} B W_{L}^{+}(s), \\
F_{L}^{-} & =(|\mathbf{k} \| \mathbf{q}|)^{L} \sqrt{\chi_{i} \chi_{f}} \frac{\alpha_{L}}{L} B W_{L}^{-}(s), \\
\chi_{i} & =m_{N}+k_{N 0}, \quad \chi_{f}=m_{N}+q_{N 0},
\end{aligned}
$$

where $L=n$ stands for ' + ' states and $L=n+1$ for '-' states.

\section{The $\gamma N$ widths and helicity amplitudes}

The decay of the state with $J=n+1 / 2$ into $\gamma N$ is described by the amplitude:

$$
\bar{\Psi}_{\alpha_{1} \ldots \alpha_{n}} V_{\alpha_{1} \ldots \alpha_{n}}^{(i \pm) \mu}\left(k^{\perp}\right) u\left(k_{N}\right) \varepsilon_{\mu}
$$

where $k_{N}$ is the momentum of the nucleon and $k^{\perp}$ is the component of the relative momentum between nucleon and photon which is orthogonal to the total momentum of the system $P\left(s=P^{2}\right)$ :

$$
\begin{gathered}
k_{\mu}^{\perp}=\frac{1}{2} \quad\left(k_{N}-k_{\gamma}\right)_{\nu} g_{\mu \nu}^{\perp}, \quad g_{\mu \nu}^{\perp}=g_{\mu \nu}-\frac{P_{\mu} P_{\nu}}{s}, \\
|\mathbf{k}|^{2}=-k_{\perp}^{2}=\frac{\left(s-m_{N}\right)^{2}}{4 s} .
\end{gathered}
$$

\subsection{The '+' states}

For the states with $n \geq 1$, three vertices can be constructed of the spin and orbital momentum operators. For '+' states the vertices are:

$$
\begin{aligned}
V_{\alpha_{1} \ldots \alpha_{n}}^{(1+) \mu}\left(k^{\perp}\right) & =\gamma_{\mu}^{\perp} i \gamma_{5} X_{\alpha_{1} \ldots \alpha_{n}}^{(n)}\left(k^{\perp}\right), \\
V_{\alpha_{1} \ldots \alpha_{n}}^{(2+) \mu}\left(k^{\perp}\right) & =\gamma_{\nu} i \gamma_{5} X_{\mu \nu \alpha_{1} \ldots \alpha_{n}}^{(n+2)}\left(k^{\perp}\right), \\
V_{\alpha_{1} \ldots \alpha_{n}}^{(3+) \mu}\left(k^{\perp}\right) & =\gamma_{\nu} i \gamma_{5} X_{\nu \alpha_{1} \ldots \alpha_{n-1}}^{(n)}\left(k^{\perp}\right) g_{\mu \alpha_{n}}^{\perp} .
\end{aligned}
$$

The first vertex is constructed using the spin $1 / 2$ operator and $L=n$ orbital momentum operator, the second one has $S=3 / 2, L=n+2$ and the third one $S=3 / 2$ and $L=n$. In case of photoproduction, the second vertex is reduced to the third one and only two amplitudes (one for 
$J=1 / 2$ ) are independent. The width factor $W^{(i, j+)}$ for the transition between vertices is expressed as follows:

$$
\begin{gathered}
F_{\nu_{1} \ldots \nu_{n}}^{\mu_{1} \ldots \mu_{n}} W_{i, j}^{+}=F_{\alpha_{1} \ldots \alpha_{n}}^{\mu_{1} \ldots \mu_{n}} \int \frac{d \Omega}{4 \pi} \tilde{V}_{\alpha_{1} \ldots \alpha_{n}}^{(i+) \mu}\left(k^{\perp}\right) \times \\
\frac{m_{N}+\hat{k}_{N}}{2 m_{N}} V_{\beta_{1} \ldots \beta_{n}}^{(j+) \nu_{n}}\left(k^{\perp}\right)\left(-g_{\mu \nu}^{\perp \perp}\right) \rho(s) F_{\nu_{1} \ldots \nu_{n}}^{\beta_{1} \ldots \beta_{n}}
\end{gathered}
$$

where $\rho(s) \equiv \rho\left(s, m_{N}, m_{\gamma}\right),\left(-g_{\mu \nu}^{\perp \perp}\right)$ describes the structure of the photon propagator:

$$
-g_{\mu \nu}^{\perp \perp}=-g_{\mu \nu}+\frac{P_{\mu} P_{\nu}}{P^{2}}+\frac{k_{\mu}^{\perp} k_{\nu}^{\perp}}{k_{\perp}^{2}} .
$$

and the operator $\tilde{V}$ differs from the operator $V$ by the ordering of $\gamma$-matrices. The width factors $W^{(i, j+)}$ for the first and third vertices are equal to:

$$
\begin{aligned}
W_{1,1}^{+} & =\frac{2 \alpha_{n}}{2 n+1}|\mathbf{k}|^{2 n} \frac{m_{N}+k_{N 0}}{2 m_{N}} \rho(s), \\
W_{3,3}^{+} & =\frac{\alpha_{n}(n+1)}{(2 n+1) n}|\mathbf{k}|^{2 n} \frac{m_{N}+k_{N 0}}{2 m_{N}} \rho(s), \\
W_{1,3}^{+} & =\frac{\alpha_{n}}{2 n+1}|\mathbf{k}|^{2 n} \frac{m_{N}+k_{N 0}}{2 m_{N}} \rho(s),
\end{aligned}
$$

where $\alpha_{n}$ is defined by (38).

If a state with total spin $J=n+1 / 2$ decays into $\gamma N$ having intrinsic spins $1 / 2$ and $3 / 2$ with couplings $g_{1}$ and $g_{3}$, the corresponding decay amplitude can be written as

$$
A_{\alpha_{1} \ldots \alpha_{n}}^{\mu(+)}=V_{\alpha_{1} \ldots \alpha_{n}}^{(1+) \mu} g_{1}(s)+V_{\alpha_{1} \ldots \alpha_{n}}^{(3+) \mu} g_{3}(s) .
$$

Then, the $\gamma N$ width is equal to:

$$
M \Gamma_{\gamma N}^{+}=W_{1,1}^{+} g_{1}^{2}(s)+2 W_{1,3}^{+} g_{1}(s) g_{3}(s)+W_{3,3}^{+} g_{3}^{2}(s) .
$$

The helicity $1 / 2$ amplitude has an operator proportional to the spin $1 / 2$ operator $V_{\alpha_{1} \ldots \alpha_{n}}^{(1+) \mu}$. The helicity $3 / 2$ operator can be constructed as a linear combination of the spin $3 / 2$ and $1 / 2$ operators, orthogonal to the $V_{\alpha_{1} \ldots \alpha_{n}}^{(1+) \mu}$ :

$$
\begin{aligned}
A_{\alpha_{1} \ldots \alpha_{n}}^{\mu(+)} & =A_{\mu ; \alpha_{1} \ldots \alpha_{n}}^{h=3 / 2}-A_{\mu ; \alpha_{1} \ldots \alpha_{n}}^{h=1 / 2} \\
A_{\mu ; \alpha_{1} \ldots \alpha_{n}}^{h=1 / 2} & =-V_{\alpha_{1} \ldots \alpha_{n}}^{(1+) \mu}\left(g_{1}(s)+\frac{1}{2} g_{3}(s)\right), \\
A_{\mu ; \alpha_{1} \ldots \alpha_{n}}^{h=3 / 2} & =\left(V_{\alpha_{1} \ldots \alpha_{n}}^{(3+) \mu}-\frac{1}{2} V_{\alpha_{1} \ldots \alpha_{n}}^{(1+) \mu}\right) g_{3}(s),
\end{aligned}
$$

where the sign " " for the helicity $1 / 2$ amplitude was introduced in accordance with the standard multipole definition. The width defined by the helicity amplitudes can be calculated using eq. (64).

$$
\begin{aligned}
& M \Gamma^{\frac{1}{2}}=\rho(s) W_{1,1}^{+}\left(g_{1}(s)+\frac{1}{2} g_{3}(s)\right)^{2}, \\
& M \Gamma^{\frac{3}{2}}=\rho(s)\left(W_{3,3}^{+}-\frac{1}{2} W_{1,3}^{+}\right) g_{3}^{2}(s) .
\end{aligned}
$$

Taking into account the standard definition of the $\gamma N$ width via helicity amplitudes,

$$
M \Gamma_{t o t}=M \Gamma^{\frac{3}{2}}+M \Gamma^{\frac{1}{2}}=\frac{\mathbf{k}^{2}}{\pi} \frac{2 m_{N}}{2 J+1}\left(\left|A_{n}^{\frac{1}{2}}\right|^{2}+\left|A_{n}^{\frac{3}{2}}\right|^{2}\right),
$$

we obtain

$$
\begin{aligned}
\left|A_{n}^{\frac{1}{2}}\right|^{2} & =\frac{\alpha_{n}(n+1)}{2 n+1} \rho(s) \pi|\mathbf{k}|^{2 n-2} \frac{\chi}{m_{N}^{2}}\left(g_{1}(s)+\frac{1}{2} g_{3}(s)\right)^{2} \\
\left|A_{n}^{\frac{3}{2}}\right|^{2} & =\alpha_{n} \rho(s) \pi|\mathbf{k}|^{2 n-2} \frac{\chi}{m_{N}^{2}} \frac{(n+2)(n+1)}{4 n(2 n+1)} g_{3}^{2}(s),
\end{aligned}
$$

where $\chi=m_{N}+k_{N 0}$.

In the case of resonance production, the vertex functions are usually normalized with certain form factors, e.g. the Blatt-Weisskopf form factors (the explicit form can be found in 36]). These form factors depend on the orbital angular momentum and radius $r$ and regularize the behaviour of the amplitude at large energies. For the '+' states the orbital momentum for both spin $1 / 2$ and $3 / 2$ operators are equal to $L=J-1 / 2=n$. Then, rewriting

$$
g_{1}(s)=\frac{g_{1 / 2}}{F\left(n,|\mathbf{k}|^{2}, r\right)}, \quad g_{3}(s)=\frac{g_{3 / 2}}{F\left(n,|\mathbf{k}|^{2}, r\right)},
$$

and, using eq.(70), the ratio of helicity amplitudes given in 36 ] is reproduced.

\subsection{The '-' states}

For the decay of a '-' state with total spin $J$ into $\gamma N$, the vertex functions have the form:

$$
\begin{aligned}
V_{\alpha_{1} \ldots \alpha_{n}}^{(1-) \mu}\left(k^{\perp}\right) & =\gamma_{\xi} \gamma_{\mu}^{\perp} X_{\xi \alpha_{1} \ldots \alpha_{n}}^{(n+1)}\left(k^{\perp}\right), \\
V_{\alpha_{1} \ldots \alpha_{n}}^{(2-) \mu}\left(k^{\perp}\right) & =X_{\mu \alpha_{1} \ldots \alpha_{n}}^{(n+1)}\left(k^{\perp}\right), \\
V_{\alpha_{1} \ldots \alpha_{n}}^{(3-) \mu}\left(k^{\perp}\right) & =X_{\alpha_{2} \ldots \alpha_{n}}^{(n-1)}\left(k^{\perp}\right) g_{\alpha_{1} \mu}^{\perp} .
\end{aligned}
$$

These vertices are constructed of the spin and orbital momentum operators with $(S=1 / 2, L=n+1),(S=3 / 2$, $L=n+1)$ and $(S=3 / 2$ and $L=n-1)$. As in case of "+" states, the second vertex provides us the same angular distribution as the third vertex. For the first and third vertices, the width factors $W_{i, j}^{-}$are equal to

$$
\begin{aligned}
& W_{1,1}^{-}=\frac{2 \alpha_{n+1}}{n+1}|\mathbf{k}|^{2 n+2} \frac{m_{N}+k_{N 0}}{2 m_{N}} \rho(s), \\
& W_{3,3}^{-}=\frac{\alpha_{n-1}(n+1)}{(2 n+1)(2 n-1)}|\mathbf{k}|^{2 n-2} \frac{m_{N}+k_{N 0}}{2 m_{N}} \rho(s), \\
& W_{1,3}^{-}=\frac{\alpha_{n-1}}{n+1}|\mathbf{k}|^{2 n} \frac{m_{N}+k_{N 0}}{2 m_{N}} \rho(s),
\end{aligned}
$$

where $\rho(s) \equiv \rho\left(s, m_{N}, m_{\gamma}\right)$.

The decay amplitude is defined by the sum of two vertices as follows:

$$
A_{\alpha_{1} \ldots \alpha_{n}}^{\mu(-)}=V_{\alpha_{1} \ldots \alpha_{n}}^{(1-) \mu} g_{1}(s)+V_{\alpha_{1} \ldots \alpha_{n}}^{(3-) \mu} g_{3}(s),
$$


and the $\gamma N$ width of the state is calculated as a sum over possible transitions:

$$
M \Gamma_{\gamma N}^{-}=W_{1,1}^{-} g_{1}^{2}(s)+2 W_{1,3}^{-} g_{1}(s) g_{3}(s)+W_{3,3}^{-} g_{3}^{2}(s) .
$$

The helicity $1 / 2$ amplitude has the operator proportional to the spin $1 / 2$ operator $V_{\alpha_{1} \ldots \alpha_{n}}^{(1+) \mu}$. The helicity $3 / 2$ operator can be constructed as a linear combination of the spin $3 / 2$ and $1 / 2$ operators orthogonal to the $V_{\alpha_{1} \ldots \alpha_{n}}^{(1+) \mu}$ :

$$
\begin{aligned}
A_{\alpha_{1} \ldots \alpha_{n}}^{\mu(-)} & =A_{\mu ; \alpha_{1} \ldots \alpha_{n}}^{h=1 / 2}-A_{\mu ; \alpha_{1} \ldots \alpha_{n}}^{h=3 / 2}, \\
A_{\mu ; \alpha_{1} \ldots \alpha_{n}}^{h=1 / 2} & =V_{\alpha_{1} \ldots \alpha_{n}}^{(1-) \mu}\left(g_{1}(s)-R g_{3}(s)\right), \\
A_{\mu ; \alpha_{1} \ldots \alpha_{n}}^{h=3 / 2} & =-\left(V_{\alpha_{1} \ldots \alpha_{n}}^{(3-) \mu}+R V_{\alpha_{1} \ldots \alpha_{n}}^{(1-) \mu}\right) g_{3}(s),
\end{aligned}
$$

where the factor $R$ is given by

$$
R=-\frac{1}{2 \mathbf{k}^{2}} \frac{\alpha_{n-1}}{\alpha_{n+1}}=-\frac{1}{2 \mathbf{k}^{2}} \frac{n(n+1)}{(2 n-1)(2 n+1)} .
$$

Here again, the signs for the helicity $1 / 2$ amplitudes are taken to correspond to the multipole definition. The widths defined by the helicity amplitudes are equal to

$$
\begin{aligned}
& M \Gamma^{\frac{1}{2}}=\rho(s) W_{1,1}^{-}\left(g_{1}(s)-R g_{3}(s)\right)^{2}, \\
& M \Gamma^{\frac{3}{2}}=\rho(s)\left(W_{3,3}^{-}+R W_{1,3}^{-}\right) g_{3}^{2}(s),
\end{aligned}
$$

and therefore

$$
\begin{aligned}
& \left|A_{n}^{\frac{1}{2}}\right|^{2}=\alpha_{n+1} \rho(s) \frac{\pi \chi}{m_{N}^{2}}|\mathbf{k}|^{2 n}\left(g_{1}(s)-R g_{3}(s)\right)^{2} \\
& \left|A_{n}^{\frac{3}{2}}\right|^{2}=\alpha_{n-1} \frac{(n+1)(n+2)}{4\left(4 n^{2}-1\right)} \rho(s) \frac{\pi \chi}{m_{N}^{2}}|\mathbf{k}|^{2 n-4} g_{3}^{2}(s)
\end{aligned}
$$

with $\chi=m_{N}+k_{N 0}$.

The vertices with couplings $g_{1}(s)$ and $g_{3}(s)$ are formed by different orbital momenta. For the state with total spin $J(n=J-1 / 2)$, the orbital momentum is equal to $L=n+1$ for the first decay $(s=1 / 2)$ and $L=n-1$ for the second one $(s=3 / 2)$. Using the Blatt-Weisskopf form factors for the normalization, we obtain

$$
g_{1}(s)=\frac{g_{1 / 2}}{F\left(n+1,|\mathbf{k}|^{2}, r\right)}, \quad g_{3}(s)=\frac{g_{3 / 2}}{F\left(n-1,|\mathbf{k}|^{2}, r\right)}
$$

\subsection{Single meson photoproduction}

General structure of the single-meson photoproduction amplitude in c.m.s. of the reaction is given by

$$
\begin{gathered}
J_{\mu}=i \mathcal{F}_{1} \sigma_{\mu}+\mathcal{F}_{2}(\sigma \mathbf{q}) \frac{\varepsilon_{\mu i j} \sigma_{i} k_{j}}{|\mathbf{k}||\mathbf{q}|}+i \mathcal{F}_{3} \frac{(\sigma \mathbf{k})}{|\mathbf{k}||\mathbf{q}|} q_{\mu}+i \mathcal{F}_{4} \frac{(\sigma \mathbf{q})}{\mathbf{q}^{2}} q_{\mu}, \\
A=\omega^{*} J_{\mu} \varepsilon_{\mu} \omega^{\prime},
\end{gathered}
$$

where $\mathbf{q}$ is the momentum of the nucleon in the $\pi N$ channel and $\mathbf{k}$ the momentum of the nucleon in the $\gamma N$ channel calculated in the c.m.s. of the reaction. The $\sigma_{i}$ are Pauli matrices.

The functions $\mathcal{F}_{i}$ have the following angular dependence:

$$
\begin{aligned}
& \mathcal{F}_{1}(z)=\sum_{L=0}^{\infty}\left[L M_{L}^{+}+E_{L}^{+}\right] P_{L+1}^{\prime}(z)+ \\
& {\left[(L+1) M_{L}^{-}+E_{L}^{-}\right] P_{L-1}^{\prime}(z),} \\
& \mathcal{F}_{2}(z)=\sum_{L=1}^{\infty}\left[(L+1) M_{L}^{+}+L M_{L}^{-}\right] P_{L}^{\prime}(z), \\
& \mathcal{F}_{3}(z)=\sum_{L=1}^{\infty}\left[E_{L}^{+}-M_{L}^{+}\right] P_{L+1}^{\prime \prime}(z)+\left[E_{L}^{-}+M_{L}^{-}\right] P_{L-1}^{\prime \prime}(z), \\
& \mathcal{F}_{4}(z)=\sum_{L=2}^{\infty}\left[M_{L}^{+}-E_{L}^{+}-M_{L}^{-}-E_{L}^{-}\right] P_{L}^{\prime \prime}(z) .
\end{aligned}
$$

Here $L$ corresponds to the orbital angular momentum in the $\pi N$ system, $P_{L}(z), P_{L}^{\prime}(z), P_{L}^{\prime \prime}(z)$ are Legendre polynomials and thier derivatives, $z=(\mathbf{k q}) /(|\mathbf{k}||\mathbf{q}|)$, and $E_{L}^{ \pm}$ and $M_{L}^{ \pm}$are electric and magnetic multipoles describing transitions to states with $J=L \pm 1 / 2$.

The single-meson production amplitude via the intermediate resonance with $J=n+1 / 2$ (we take pion photoproduction as an example) has the general form:

$$
\begin{aligned}
A^{i \pm} & =g_{\pi N}(s) \bar{u}\left(q_{N}\right) \tilde{N}_{\alpha_{1} \ldots \alpha_{n}}^{ \pm}\left(q^{\perp}\right) \times \\
& \frac{F_{\beta_{1} \ldots \beta_{n}}^{\alpha_{1} \ldots \alpha_{n}}}{M^{2}-s-i M \Gamma_{t o t}} V_{\beta_{1} \ldots \beta_{n}}^{(i \pm) \mu}\left(k^{\perp}\right) u\left(k_{N}\right) g_{i}(s) \varepsilon_{\mu} .
\end{aligned}
$$

Here, $q_{N}$ and $k_{N}$ are the momenta of the nucleon in the $\pi N$ and $\gamma N$ channel and $q^{\perp}$ and $k^{\perp}$ are the components of relative momenta which are orthogonal to the total momentum of the resonance. The index $i$ lists the $\gamma N$ vertices given in (61), (72).

The multipole decomposition of the amplitude is given in detail in 36]. Below we give the final expressions for

$$
E_{L}^{ \pm}=E_{L}^{ \pm\left(\frac{1}{2}\right)}+E_{L}^{ \pm\left(\frac{3}{2}\right)} \quad M_{L}^{ \pm}=M_{L}^{ \pm\left(\frac{1}{2}\right)}+M_{L}^{ \pm\left(\frac{3}{2}\right)} .
$$

For the '+' states (where $L=n$ ):

$$
\begin{aligned}
E_{L}^{+\left(\frac{1}{2}\right)} & =\frac{\sqrt{\chi_{i} \chi_{f}} \alpha_{L}}{(2 L+1)(L+1)} \frac{g_{\pi N}(s)(|\mathbf{k}||\mathbf{q}|)^{L} g_{1}(s)}{M^{2}-s-i M \Gamma_{t o t}} \\
M_{L}^{+\left(\frac{1}{2}\right)} & =E_{L}^{+\left(\frac{1}{2}\right)}, \\
E_{L}^{+\left(\frac{3}{2}\right)} & =\frac{\sqrt{\chi_{i} \chi_{f}} \alpha_{L}}{(2 L+1)(L+1)} \frac{g_{\pi N}(s)(|\mathbf{k}||\mathbf{q}|)^{L} g_{3}(s)}{M^{2}-s-i M \Gamma_{t o t}} \\
M_{L}^{+\left(\frac{3}{2}\right)} & =-\frac{E_{L}^{+\left(\frac{3}{2}\right)}}{L} .
\end{aligned}
$$

Remember that $\chi_{i}=m_{N}+q_{N 0}$ and $\chi_{f}=m_{N}+k_{N 0}$. For the '-' states, where $L=n+1$, the corresponding equations are

$$
\begin{aligned}
E_{L}^{-\left(\frac{1}{2}\right)} & =-\sqrt{\chi_{i} \chi_{f}} \frac{\alpha_{L}}{L^{2}} \frac{g_{\pi N}(|\mathbf{k}||\mathbf{q}|)^{L} g_{1}(s)}{M^{2}-s-i M \Gamma_{t o t}} \\
M_{L}^{-\left(\frac{1}{2}\right)} & =-E_{L}^{-\left(\frac{1}{2}\right)} \\
E_{L}^{-\left(\frac{3}{2}\right)} & =-\frac{\alpha_{L-2}}{(L-1) L} \sqrt{\chi_{i} \chi_{f}} \frac{g_{\pi N}|\mathbf{k}|^{L-2}|\mathbf{q}|^{L} g_{3}(s)}{M^{2}-s-i M \Gamma_{t o t}} \\
M_{L}^{-\left(\frac{3}{2}\right)} & =0
\end{aligned}
$$


These formulae are different from the correspondent expressions given in [36] by the factor $(-1)^{n}$ which enters now in the resonance propagator. All other formulae given in 36] for the single meson photoproduction are not changed due to this redefinition.

\section{Three-body partial widths of the baryon resonances}

\subsection{Three-body final states}

The total width of the state is calculated by averaging over polarizations of the resonance and summing over polarizations of the final-state particles. For the three-particle final state, the amplitude squared depends on three invariants, total momentum squared and two intermediate momenta squared $s_{i j}=\left(q_{i}+q_{j}\right)^{2}$. After performing the integration over other variables we obtain

$$
\frac{(2 \pi)^{4}}{2} d \Phi_{3}\left(P, q_{1}, q_{2}, q_{3}\right)=\int \frac{1}{32 s(2 \pi)^{3}} d s_{12} d s_{23} .
$$

The integration limits for $s_{12}$ are $\left(m_{1}+m_{2}\right)^{2}$ and $(\sqrt{s}-$ $\left.m_{3}\right)^{2}$. For given value of $s_{12}$, the limitsfor $s_{23}$ are defined as follows:

$$
\begin{gathered}
s_{23}^{ \pm}=\left(E_{2}+E_{3}\right)^{2}-\left(\sqrt{E_{2}^{2}-m_{2}^{2}} \pm \sqrt{E_{3}^{2}-m_{3}^{2}}\right)^{2} \\
E_{2}=\frac{s_{12}-m_{1}^{2}+m_{2}^{2}}{2 \sqrt{s_{12}}}, \quad E_{3}=\frac{s-s_{12}-m_{3}^{2}}{2 \sqrt{s_{12}}} .
\end{gathered}
$$

The three body phase space can also be written as a product of the two two-body phase spaces, e.g. the phase space of particles 1,2 and the phase space of the $(1,2)$ system and the third particle:

$$
\begin{aligned}
& \frac{(2 \pi)^{4}}{2} d \Phi\left(P, q_{1}, q_{2}, q_{3}\right)=\frac{(2 \pi)^{4}}{2} d \Phi\left(q_{1}+q_{2}, q_{1}, q_{2}\right) \times \\
& \frac{(2 \pi)^{4}}{2} d \Phi\left(P, q_{1}+q_{2}, q_{3}\right) \frac{d s_{12}}{\pi} .
\end{aligned}
$$

This expression is very useful for the cascade decays when a resonance is accompanied by a 'spectator' particle and then decays into two particles. Let us write the explicit form of the expression $Q \otimes Q$ for the width of baryon with spin $J(n=J-1 / 2)$ which decays into a nucleon with momentum $q_{3} \equiv q_{N}$ and a meson resonance which decays subsequently into two mesons with momenta $q_{1}$ and $q_{2}$ (we denote them as $\pi$ and $\eta$ ). If the spin of the intermediate resonance is $J_{12}\left(J_{12}=m\right)$, its decay into two pseudoscalar mesons is described by the orbital momentum $X^{(m)}$, and therefore

$$
\begin{aligned}
& Q_{\mu_{1} \ldots \mu_{n}} \otimes Q_{\nu_{1} \ldots \nu_{n}}=\tilde{P}_{\mu_{1} \ldots \mu_{n}}^{\alpha_{1} \ldots \alpha_{m}} \frac{m_{N}+\hat{q}_{N}}{2 m_{N}} \times \\
& \frac{f_{\beta_{1} \ldots \beta_{m}}^{\alpha_{1} \ldots \alpha_{m}} g\left(s_{12}\right)}{M_{R}^{2}-s_{12}-i M_{R} \Gamma_{t o t}^{R}} X_{\beta_{1} \ldots \beta_{m}}^{(m)}\left(q_{12}^{\perp}\right) X_{\xi_{1} \ldots \xi_{m}}^{(m)}\left(q_{12}^{\perp}\right) \times \\
& \frac{g\left(s_{12}\right) f_{\eta_{1} \ldots \eta_{m}}^{\xi_{1} \ldots \xi_{m}}}{M_{R}^{2}-s_{12}+i M_{R} \Gamma_{t o t}^{R}} P_{\eta_{1} \ldots \eta_{n}}^{\nu_{1} \ldots \nu_{m}} .
\end{aligned}
$$

Here we denote the mass and the total width of the inter-

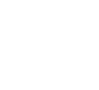

.

\section{.}

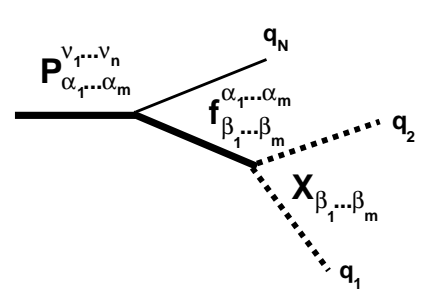

Fig. 2. Resonance decay into nucleon and two meson states

the intermediate resonance to the final-state mesons; the operator $P$ describes the decay of the initial state into resonance $R$ and spectator nucleon. The operator $\tilde{P}$ differs from the operator $P$ by the permutation of $\gamma$-matrices. The $q \frac{\perp}{12}$ is the relative momentum of the mesons:

$$
\begin{aligned}
& \tilde{g}_{\mu \nu}^{\perp}=g_{\mu \nu}-\frac{k_{\mu} k_{\nu}}{s_{12}}, \quad k=q_{1}+q_{2}, \\
& q_{12 \mu}^{\perp}=\frac{1}{2}\left(q_{1}-q_{2}\right)_{\nu} \tilde{g}_{\mu \nu}^{\perp} .
\end{aligned}
$$

Using basic equation (11) for the width

$$
\begin{gathered}
f_{\eta_{1} \ldots \eta_{m}}^{\alpha_{1} \ldots \alpha_{m}} M_{R} \Gamma_{\pi \eta}^{R}=\int \frac{(2 \pi)^{4}}{2} d \Phi\left(k, q_{1}, q_{2}\right) f_{\beta_{1} \ldots \beta_{m}}^{\alpha_{1} \ldots \alpha_{m}} \times \\
g\left(s_{12}\right) X_{\beta_{1} \ldots \beta_{m}}\left(q_{12}^{\perp}\right) X_{\xi_{1} \ldots \xi_{m}}\left(q_{12}^{\perp}\right) g\left(s_{12}\right) f_{\eta_{1} \ldots \eta_{m}}^{\xi_{1} \ldots \xi_{m}}
\end{gathered}
$$

we obtain the final expression for the width of the initial resonance

$$
\begin{array}{r}
F_{\beta_{1} \ldots \beta_{n}}^{\alpha_{1} \ldots \alpha_{n}} M \Gamma=F_{\mu_{1} \ldots \mu_{n}}^{\alpha_{1} \ldots \alpha_{n}} \int \frac{d s_{12}}{\pi} \frac{(2 \pi)^{4}}{2} d \Phi\left(P, k, q_{3}\right) \times \\
g^{2}(s) \tilde{P}_{\mu_{1} \ldots \mu_{n}}^{\xi_{1} \ldots \xi_{m}} \frac{f_{\eta_{1} \ldots \eta_{m}}^{\xi_{1} \ldots \xi_{m}} M_{R} \Gamma_{\pi \eta}^{R}}{\left(M_{R}^{2}-s\right)^{2}+\left(M_{R} \Gamma_{t o t}^{R}\right)^{2}} P_{\nu_{1} \ldots \nu_{n}}^{\eta_{1} \ldots \eta_{m}} \cdot F_{\beta_{1} \ldots \beta_{n}}^{\nu_{1} \ldots \nu_{n}} .(93
\end{array}
$$

In the limit of zero width of the intermediate state we have

$$
\int \frac{d s_{12}}{\pi} \frac{M_{R} \Gamma_{t o t}^{R}}{\left(M_{R}^{2}-s_{12}\right)^{2}+\left(M_{R} \Gamma_{t o t}^{R}\right)^{2}}=\int d s_{12} \delta\left(M_{R}^{2}-s_{12}\right)(
$$

and equation (93) is reduced to the two-body equation multiplied by the branching ratio of the decay of the intermediate state, $B r_{\pi \eta}=\Gamma_{\pi \eta}^{R} / \Gamma_{t o t}^{R}$.

Let us note that provided a resonance has many decay modes (or the mode can be in different kinematical channels), the decay amplitude can be written as a vector with components corresponding to these decay modes. In this case, eq. (93) gives us diagonal transition elements only. To obtain nondiagonal elements between different kinematical channels, it is necessary to use general expression for the phase volume (87). 

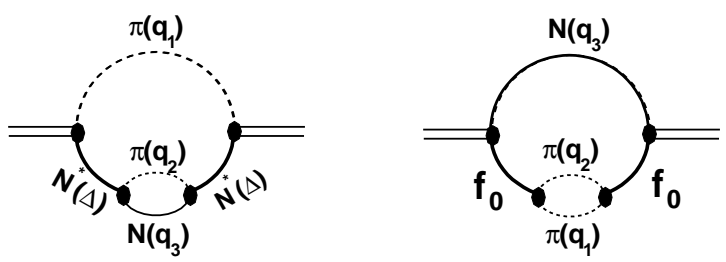

Fig. 3. $\pi N^{*}(\pi \Delta)$ and $f_{0} N$ loop diagrams

\section{$6.2 f_{0} N$ partial widths of the baryon resonances}

Let us start with the calculation of the $f_{0} N$ loop diagram where $f_{0}$ denotes a scalar resonance which decays into two pseudoscalar mesons with momenta $q_{1}$ and $q_{2}$ (see fig. 33). The width of the scalar state is defined by

$$
M_{R} \Gamma_{\pi \pi}^{R}=g_{\pi \pi}^{2}\left(s_{12}\right) \rho\left(s_{12}, m_{1}, m_{2}\right)
$$

Here, $g_{\pi \pi}$ is the coupling of the resonance into two pseudoscalar mesons. For the simplicity sake, we denote them as pions but use different masses $m_{1}$ and $m_{2}$. Thus, the final expressions can be directly used for the decay of any scalar state into two pseudoscalar particles.

The decay of the baryon state into scalar meson and nucleon is described by the same vertices as the decay of a baryon into $\pi N$. The only difference is that due to the positive parity of the scalar meson, the decay of ' + ' baryons will be defined by $N_{\mu_{1} \ldots \mu_{n}}^{-}$operators and decay of the '-' baryons by the $N_{\mu_{1} \ldots \mu_{n}}^{+}$operators:

$$
\begin{aligned}
& P_{\mu_{1} \ldots \mu_{n}}^{(+)}=N_{\mu_{1} \ldots \mu_{n}}^{-}, \\
& P_{\mu_{1} \ldots \mu_{n}}^{(-)}=N_{\mu_{1} \ldots \mu_{n}}^{+} .
\end{aligned}
$$

Thus, we can use the results given in eqs. (55156) to obtain

$$
M \Gamma_{f_{0} N}^{ \pm}=\int_{\left(m_{1}+m_{2}\right)^{2}}^{\left(\sqrt{s}-m_{3}\right)^{2}} \frac{d s_{12}}{\pi} \frac{\rho\left(s, \sqrt{s_{12}}, m_{N}\right) W^{ \pm} M_{R} \Gamma_{\pi \pi}^{R}}{\left(M_{R}^{2}-s\right)^{2}+\left(M_{R} \Gamma_{t o t}^{R}\right)^{2}}
$$

where

$$
\begin{aligned}
W^{(+)} & =\frac{\alpha_{n+1}}{n+1}\left|\mathbf{q}_{N}\right|^{2 n+2} \frac{m_{N}+q_{N 0}}{2 m_{N}} g_{f_{0} N}^{2}(s), \\
W^{(-)} & =\frac{\alpha_{n}}{2 n+1}\left|\mathbf{q}_{N}\right|^{2 n} \frac{m_{N}+q_{N 0}}{2 m_{N}} g_{f_{0} N}^{2}(s),
\end{aligned}
$$

and

$$
q_{N 0}=\frac{s+m_{N}^{2}-s_{12}}{2 \sqrt{s}}, \quad\left|\mathbf{q}_{N}\right|^{2}=q_{N 0}^{2}-m_{N}^{2} .
$$

\subsection{Vector-meson $-N$ partial widths of baryon resonances}

The decay vertex of the vector particle into two pseudoscalar particles is defined by the operator $X_{\mu}^{(1)}$, and the width is equal to

$$
\begin{gathered}
M_{R} \Gamma_{\pi \pi}^{R}=g_{\pi \pi}^{2}\left(s_{12}\right) \frac{\left|\mathbf{q}_{\pi}\right|^{2}}{3} \rho\left(s_{12}, m_{1}, m_{2}\right), \\
\left|\mathbf{q}_{\pi}\right|^{2}=\frac{\left(s_{12}-\left(m_{1}+m_{2}\right)^{2}\right)\left(s_{12}-\left(m_{1}-m_{2}\right)^{2}\right)}{4 s_{12}} .
\end{gathered}
$$

Here, $g_{\pi \pi}$ is the coupling of the vector meson into two pseudoscalar mesons with masses $m_{1}$ and $m_{2}$.

The decay of the baryon into vector meson and nucleon is described by the same vertices as the decay of baryons into $\gamma N$. In this case, all three vertices are independent and the width is formed by all possible transitions between vertices:

$$
\begin{aligned}
& M \Gamma_{\rho N}^{ \pm}=\int_{\left(m_{1}+m_{2}\right)^{2}}^{\left(\sqrt{s}-m_{N}\right)^{2}} \frac{d s_{12}}{\pi} \frac{\rho\left(s, \sqrt{s_{12}}, m_{N}\right) W^{ \pm} M_{R} \Gamma_{\pi \pi}^{R}}{\left(M_{R}^{2}-s\right)^{2}+\left(M_{R} \Gamma_{t o t}^{R}\right)^{2}} \\
& W^{ \pm}=\sum_{i, j=1}^{3} W_{i, j}^{ \pm} g_{i}(s) g_{j}^{*}(s)
\end{aligned}
$$

where we assume that couplings can be complex magnitudes and the $W_{i, j}^{ \pm}$functions are defined as follows:

$$
\begin{aligned}
F_{\beta_{1} \ldots \beta_{n}}^{\alpha_{1} \ldots \alpha_{n}} W_{i, j}^{ \pm}= & F_{\mu_{1} \ldots \mu_{n}}^{\alpha_{1} \ldots \alpha_{n}} \int \frac{d \Omega}{4 \pi} \tilde{V}_{\mu_{1} \ldots \mu_{n}}^{(i \pm) \xi} \frac{m_{N}+\hat{q}_{N}}{2 m_{N}} \times \\
& \left(\frac{k_{\xi} k_{\eta}}{s_{12}}-g_{\xi \eta}\right) V_{\nu_{1} \ldots \nu_{n}}^{(i \pm) \eta} F_{\beta_{1} \ldots \beta_{n}}^{\nu_{1} \ldots \nu_{n}} .
\end{aligned}
$$

The vertex functions $V^{(i \pm)}$ are given by eqs. (6172) and $k=q_{1}+q_{2}$.

For the '+' states the $W$ functions are given by

$$
\begin{aligned}
& W_{1,1}^{+}=\frac{\alpha_{n}}{2 n+1}\left|\mathbf{q}_{N}\right|^{2 n} \frac{m_{N}+q_{N 0}}{2 m_{N}}\left(3+\frac{\left|\mathbf{q}_{N}\right|^{2}}{s_{12}}\right), \\
& W_{1,2}^{+}=-\frac{\alpha_{n}}{n+1} \frac{\left|\mathbf{q}_{N}\right|^{2 n+4}}{s_{12}} \frac{m_{N}+q_{N 0}}{2 m_{N}}, \\
& W_{1,3}^{+}=\frac{\alpha_{n}}{2 n+1}\left|\mathbf{q}_{N}\right|^{2 n} \frac{m_{N}+q_{N 0}}{2 m_{N}}\left(2+\frac{\left|\mathbf{q}_{N}\right|^{2}}{s_{12}}\right), \\
& W_{2,2}^{+}=\frac{\alpha_{n+1}\left|\mathbf{q}_{N}\right|^{2 n+4}}{n+1} \frac{m_{N}+q_{N 0}}{2 m_{N}}\left(\frac{2 n+3}{n+2}+\frac{\left|\mathbf{q}_{N}\right|^{2}}{s_{12}}\right), \\
& W_{2,3}^{+}=-\frac{\alpha_{n+1}}{2 n+1} \frac{\left|\mathbf{q}_{N}\right|^{2 n+4}}{s_{12}} \frac{m_{N}+q_{N 0}}{2 m_{N}}, \\
& W_{3,3}^{+}=\frac{\alpha_{n}\left|\mathbf{q}_{N}\right|^{2 n}}{n} \frac{m_{N}+q_{N 0}}{2 m_{N}}\left(1+\frac{n\left|\mathbf{q}_{N}\right|^{2}}{(2 n+1) s_{12}}\right),
\end{aligned}
$$

and the '-' states by

$$
W_{1,1}^{-}=\frac{\alpha_{n+1}}{n+1}\left|\mathbf{q}_{N}\right|^{2 n+2} \frac{m_{N}+q_{N 0}}{2 m_{N}}\left(3+\frac{\left|\mathbf{q}_{N}\right|^{2}}{s_{12}}\right),
$$




$$
\begin{aligned}
& W_{1,2}^{-}=\frac{\left|\mathbf{q}_{N}\right|^{2 n+2}}{n+1} \frac{m_{N}+q_{N 0}}{2 m_{N}}\left(\alpha_{n+1}+\alpha_{n} \frac{\left|\mathbf{q}_{N}\right|^{2}}{s_{12}}\right) \\
& W_{1,3}^{-}=-\frac{\alpha_{n-1}}{n+1} \frac{\left|\mathbf{q}_{N}\right|^{2 n+2}}{s_{12}} \frac{m_{N}+q_{N 0}}{2 m_{N}} \\
& W_{2,2}^{-}=\frac{\left|\mathbf{q}_{N}\right|^{2 n+2}}{2 n+1} \frac{m_{N}+q_{N 0}}{2 m_{N}}\left(\alpha_{n+1}+\alpha_{n} \frac{\left|\mathbf{q}_{N}\right|^{2}}{s_{12}}\right), \\
& W_{2,3}^{-}=-\frac{\alpha_{n-1}}{2 n+1} \frac{\left|\mathbf{q}_{N}\right|^{2 n+2}}{s_{12}} \frac{m_{N}+q_{N 0}}{2 m_{N}}, \\
& W_{3,3}^{-}=\frac{\alpha_{n-1}\left|\mathbf{q}_{N}\right|^{2 n-2}}{2 n-1} \frac{m_{N}+q_{N 0}}{2 m_{N}}\left(1+\frac{n\left|\mathbf{q}_{N}\right|^{2}}{(2 n+1) s_{12}}\right)
\end{aligned}
$$

The $q_{N 0}$ and $\left|\mathbf{q}_{N}\right|$ are defined by eq. (99).

\subsection{The $\pi P_{11}$ and $\pi S_{11}$ partial widths of baryon resonances}

Let us calculate the partial width of the baryon resonance decaying into pseudoscalar meson and $P_{11}$ state (e.g. the Roper resonance) which in turn decays into pseudoscalar meson and nucleon with the momenta $q_{2}$ and $q_{3} \equiv q_{N}$ (see fig (3). The partial width of the $P_{11}$ state is defined by the equation (56) at $n=0$. Therefore,

$$
M \Gamma^{ \pm}=\int_{\left(m_{2}+m_{3}\right)^{2}}^{\left(\sqrt{s}-m_{1}\right)^{2}} \frac{d s_{23}}{\pi} \frac{\rho\left(s, \sqrt{s_{23}}, m_{N}\right) W^{ \pm} M_{R} \Gamma_{\pi N}^{R}}{\left(M_{R}^{2}-s_{23}\right)^{2}+\left(M_{R} \Gamma_{t o t}^{R}\right)^{2}},
$$

where

$$
\begin{aligned}
M_{R} \Gamma_{\pi N}^{R} & =\left|\mathbf{q}_{N}\right|^{2} \frac{m_{N}+q_{N 0}}{2 m_{N}} \rho\left(s_{23}, m_{2}, m_{N}\right) g_{\pi N}^{2}\left(s_{23}\right), \\
q_{N 0} & =\frac{s_{23}+m_{N}^{2}-m_{2}^{2}}{2 \sqrt{s_{23}}}, \quad\left|\mathbf{q}_{N}\right|^{2}=q_{N 0}^{2}-m_{N}^{2},
\end{aligned}
$$

and $W^{ \pm}$are defined by eqs. (55156). The mass and momentum of the nucleon should be substituted by the running mass $\sqrt{s_{23}}$ and momentum of the $P_{11}$ state $k$ :

$$
\begin{aligned}
& W^{+}=\frac{\alpha_{n}}{2 n+1}|\mathbf{k}|^{2 n} \frac{k_{0}+\sqrt{s_{23}}}{2 \sqrt{s_{23}}} g^{2}(s), \\
& W^{-}=\frac{\alpha_{n+1}}{n+1}|\mathbf{k}|^{2 n+2} \frac{k_{0}+\sqrt{s_{23}}}{2 \sqrt{s_{23}}} g^{2}(s),
\end{aligned}
$$

with

$$
k_{0}=\frac{s+s_{23}-m_{1}}{2 \sqrt{s}}, \quad|\mathbf{k}|^{2}=k_{0}^{2}-s_{23} .
$$

The partial width of the $S_{11}$ state into $\pi N$ is defined by eq. (55) with $n=0$ :

$$
M_{R} \Gamma_{\pi N}^{R}=\frac{m_{N}+q_{N 0}}{2 m_{N}} \rho\left(s_{23}, m_{2}, m_{N}\right) g_{\pi N}^{2}\left(s_{23}\right) .
$$

The baryon decay into $\pi S_{11}$ differs from the decay into $\pi P_{11}$ by $\mathrm{P}$-parity and can be calculated by substituting $N^{ \pm}$operators by $N^{\mp}$ operators. Thus,

$$
\begin{aligned}
& W^{+}=\frac{\alpha_{n+1}}{n+1}|\mathbf{k}|^{2 n+2} \frac{k_{0}+\sqrt{s_{23}}}{2 \sqrt{s_{23}}} g^{2}(s), \\
& W^{-}=\frac{\alpha_{n}}{2 n+1}|\mathbf{k}|^{2 n} \frac{k_{0}+\sqrt{s_{23}}}{2 \sqrt{s_{23}}} g^{2}(s) .
\end{aligned}
$$

\subsection{The $\pi \Delta\left(3 / 2^{+}\right)$partial widths of the baryon resonances}

The baryon partial width into $\Delta \pi$, where the $\Delta$ resonance is produced in the channel (23), can be written as follows:

$$
M \Gamma^{ \pm}=\int_{\left(m_{2}+m_{3}\right)^{2}}^{\left(\sqrt{s}-m_{1}\right)^{2}} \frac{d s_{23}}{\pi} \frac{\rho\left(s, \sqrt{s_{23}}, m_{N}\right) W^{ \pm} M_{R} \Gamma_{\pi N}^{R}}{\left(M_{R}^{2}-s_{23}\right)^{2}+\left(M_{R} \Gamma_{t o t}^{R}\right)^{2}} .
$$

The partial width of the $\frac{3}{2}^{+}$state is defined by eq.(55) with $\mathrm{n}=1$ :

$$
\begin{gathered}
M_{R} \Gamma_{\pi N}^{R}=\frac{\left|\mathbf{q}_{N}\right|^{2}}{3} \frac{m_{N}+q_{N 0}}{2 m_{N}} \rho\left(s_{23}, m_{2}, m_{3}\right) g_{\pi N}^{2}\left(s_{23}\right), \\
q_{N 0}=\frac{s_{23}+m_{N}^{2}-m_{2}^{2}}{2 \sqrt{s}_{23}} \quad\left|\mathbf{q}_{N}\right|^{2}=q_{N 0}^{2}-m_{N}^{2} .
\end{gathered}
$$

The decay of baryons with the total spin $J \geq 3 / 2$ into pseudoscalar meson and baryon with spin $3 / 2$ has two vertices which are defined by different combinations of spins in the intermediate state and the orbital momentum between this state and a spectator particle. The decay of ' + ' states is now described by the follows operators

$$
\begin{aligned}
& P_{\alpha_{1} \ldots \alpha_{n}}^{(1+) \mu} \Psi_{\mu}=i \gamma_{5} \gamma_{\nu} X_{\mu \nu \alpha_{1} \ldots \alpha_{n}}^{(n+2)} \Psi_{\mu}, \\
& P_{\alpha_{1} \ldots \alpha_{n}}^{(2+) \mu} \Psi_{\mu}=i \gamma_{5} \gamma_{\nu} X_{\nu \alpha_{2} \ldots \alpha_{n}}^{(n)} g_{\alpha_{1} \mu}^{\perp} \Psi_{\mu} .
\end{aligned}
$$
by

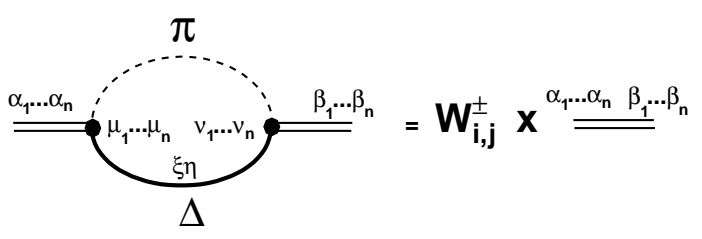

Fig. 4. Index definition in the $\pi \Delta$ loop diagram

The operators for the decay of the '-' states are given

$$
\begin{aligned}
& P_{\alpha_{1} \ldots \alpha_{n}}^{(1-) \Psi_{\mu}} \Psi_{\mu}=X_{\mu \alpha_{1} \ldots \alpha_{n}}^{(n+1)} \Psi_{\mu}, \\
& P_{\alpha_{1} \ldots \alpha_{n}}^{(2-) \mu} \Psi_{\mu}=X_{\alpha_{2} \ldots \alpha_{n}}^{(n-1)} g_{\alpha_{1} \mu}^{\perp} \Psi_{\mu} .
\end{aligned}
$$

The $W^{ \pm}$functions are defined by the squared sum of the decay amplitudes

$$
W^{ \pm}=\sum_{i, j=1}^{2} W_{i, j}^{ \pm} g_{i}(s) g_{j}^{*}(s),
$$

where $W_{i, j}^{ \pm}$are defined as follows (see fig 4 for the index definition):

$$
\begin{gathered}
F_{\beta_{1} \ldots \beta_{n}}^{\alpha_{1} \ldots \alpha_{n}} W_{i, j}^{ \pm}=F_{\mu_{1} \ldots \mu_{n}}^{\alpha_{1} \ldots \alpha_{n}} \int \frac{d \Omega}{4 \pi} \tilde{P}_{\mu_{1} \ldots \mu_{n}}^{(i \pm) \xi} \times \\
\left(\frac{\gamma_{\xi}^{\perp} \gamma_{\eta}^{\perp}}{3}-\tilde{g}_{\xi \eta}^{\perp}\right) \frac{\sqrt{s_{23}}+\hat{k}}{2 \sqrt{s_{23}}} P_{\nu_{1} \ldots \nu_{n}}^{(i \pm) \eta} F_{\beta_{1} \ldots \beta_{n}}^{\nu_{1} \ldots \nu_{n}}
\end{gathered}
$$


Here, $k=q_{2}+q_{3}$, and the metric tensor orthogonal to the momentum of $\Delta$ is equal to

$$
\tilde{g}_{\xi \eta}^{\perp}=g_{\xi \eta}-\frac{k_{\xi} k_{\eta}}{s_{23}}, \quad \gamma_{\mu}^{\perp}=\gamma_{\nu} \tilde{g}_{\mu \nu}^{\perp}
$$

It is useful to extract from the numerator of the $\Delta$ propagator the nonorthogonal part

$$
\frac{\gamma_{\xi} \gamma_{\eta}}{3}-g_{\xi \eta}
$$

Then, the equation for $W_{i, j}^{ \pm}$can be rewritten as follows:

$$
\begin{gathered}
W_{i, j}^{ \pm}=Z_{i, j}^{(1 \pm)}+\frac{|\mathbf{k}|^{2}}{3 s_{23}} Z_{i, j}^{(2 \pm)}, \\
k_{0}=\frac{s+s_{23}-m_{1}}{2 \sqrt{s}}, \quad|\mathbf{k}|^{2}=k_{0}^{2}-s_{23},
\end{gathered}
$$

where $Z_{i, j}^{(1)}$ is defined as a contribution from (118), and the rest is the difference between a full expression and eq. (118). The second part should be proportional to the momentum of $\Delta$ which we extracted in explicit form. In the decay of the baryon with the mass not far from the $\Delta \pi$ threshold the contribution of this part should be much smaller than the contribution of the nonorthogonal part.

After some cumbersome calculations (an example is given in Appendix), we obtain for the '+' states

$$
\begin{aligned}
& Z_{1,1}^{(1+)}=|\mathbf{k}|^{2(n+2)} \frac{\alpha_{n+2}}{n+1} \frac{\sqrt{s_{23}}+k_{0}}{2 \sqrt{s_{23}}}, \\
& Z_{1,1}^{(2+)}=|\mathbf{k}|^{2(n+2)} \frac{\alpha_{n+1}}{n+1} \frac{2 \sqrt{s_{23}}+k_{0}}{\sqrt{s_{23}}}, \\
& Z_{2,2}^{(1+)}=|\mathbf{k}|^{2 n} \frac{\alpha_{n}(2 n+3)}{3 n(2 n+1)} \frac{\sqrt{s_{23}}+k_{0}}{2 \sqrt{s_{23}}}, \\
& Z_{2,2}^{(2+)}=|\mathbf{k}|^{2 n} \frac{\alpha_{n}}{2 n+1} \frac{k_{0}}{\sqrt{s_{23}}}, \\
& Z_{1,2}^{(1+)}=0, \\
& Z_{1,2}^{(2+)}=-|\mathbf{k}|^{2(n+1)} \frac{\alpha_{n+1}}{2 n+1} \frac{\sqrt{s_{23}}+k_{0}}{\sqrt{s_{23}}},
\end{aligned}
$$

and for the '-' states

$$
\begin{aligned}
& Z_{1,1}^{(1-)}=|\mathbf{k}|^{2(n+1)} \frac{\alpha_{n+1}(n+2)}{3(n+1)(2 n+1)} \frac{\sqrt{s_{23}}+k_{0}}{2 \sqrt{s_{23}}}, \\
& Z_{1,1}^{(2-)}=|\mathbf{k}|^{2(n+1)} \frac{\alpha_{n}}{2 n+1} \frac{\sqrt{s_{23}}+k_{0}(n+1)}{\sqrt{s_{23}}(n+1)}, \\
& Z_{2,2}^{(1-)}=|\mathbf{k}|^{2(n-1)} \frac{\alpha_{n-1}}{2 n-1} \frac{\sqrt{s_{23}}+k_{0}}{2 \sqrt{s_{23}}}, \\
& Z_{2,2}^{(2-)}=|\mathbf{k}|^{2(n-1)} \frac{\alpha_{n-1} n}{(2 n-1)(2 n+1)} \frac{2 \sqrt{s_{23}}+k_{0}}{\sqrt{s_{23}}}, \\
& Z_{1,2}^{(1-)}=0, \\
& Z_{1,2}^{(2-)}=-|\mathbf{k}|^{2 n} \frac{\alpha_{n-1}}{2 n+1}\left(\frac{\sqrt{s_{23}}+k_{0}}{\sqrt{s_{23}}}+\frac{1}{2(n+1)}\right)
\end{aligned}
$$

\subsection{The $\pi 3 / 2^{-}$partial widths of baryon resonances}

In the same way, one can calculate partial widths for the resonance decay into $\pi 3 / 2^{-}$states $\left(\pi D_{13}\right.$ or $\left.\pi D_{33}\right)$. The partial width of the $\frac{3}{2}^{-}$state (which belongs to the '-' states) is defined by eq. (56) with $\mathrm{n}=1$ :

$$
M_{R} \Gamma_{\pi N}^{R}=\frac{3\left|\mathbf{q}_{N}\right|^{4}}{4} \frac{m_{N}+q_{N 0}}{2 m_{N}} \rho\left(s_{23}, m_{2}, m_{3}\right) g_{\pi N}^{2}\left(s_{23}\right)
$$

The decay of the initial-state baryon into $\pi 3 / 2^{-}$differs from the $\pi 3 / 2^{+}$decay by the $\mathrm{P}$-parity requiring the substitution of $W^{ \pm}$by $W^{\mp}$ :

$$
\begin{gathered}
W_{i, j}^{+}\left(\pi 3 / 2^{-}\right)=W_{i, j}^{-}\left(\pi 3 / 2^{+}\right), \\
W_{i, j}^{-}\left(\pi 3 / 2^{-}\right)=W_{i, j}^{+}\left(\pi 3 / 2^{+}\right) .
\end{gathered}
$$

\subsection{Singularities in the cascade decay}

The one of the most important point in the partial wave analysis is to define the position of amplitude singularities in the complex plane of the energy squared. Resonances correspond to pole singularities of the transition amplitude, while the production of the two or more particles corresponds to threshold singularities. Every threshold singularity creates two sheets in the complex plane $s$. In case of the two particle amplitude, these sheets may be reached by different paths: either directly down to the complex plane or around threshold singularity. For the resonance cascade decay into three particles, the structure is more complicated, and different sheets may be reached by the choice of the integral path in the analytical expressions for the resonance partial widths given above.

Consider as an example the structure of the singularities in the decay of a resonance into the $\pi \Delta(1232)$ channel. The imaginary part of the Breit-Wigner pole is defined by the discontinuity of the diagram shown in Fig. 3

$$
\frac{g_{\pi \Delta}^{2}}{M^{2}-s-i g_{\pi \Delta}^{2} \rho_{3}(s)}
$$

If the intermediate resonance decays into particles 2 and 3 (with momenta $k_{2}$ and $k_{3}, s_{23}=\left(k_{2}+k_{3}\right)^{2}$ ), the threebody phase volume $\rho_{3}(s)$ is defined as follows:

$$
\begin{gathered}
\rho_{3}(s)=\int_{\left(m_{2}+m_{3}\right)^{2}}^{\left(\sqrt{s}-m_{1}\right)^{2}} \frac{d s_{23}}{\pi} I\left(s, s_{23}\right), \\
I\left(s, s_{23}\right)=\frac{\rho\left(s, \sqrt{s_{23}}, m_{1}\right) M_{R} \Gamma_{t o t}^{R}}{\left(M_{R}^{2}-s_{23}\right)^{2}+\left(M_{R} \Gamma_{t o t}^{R}\right)^{2}}, \\
M_{R} \Gamma_{t o t}^{R}=\rho\left(s_{23}, m_{2}, m_{3}\right) g^{2}\left(s_{23}\right),
\end{gathered}
$$

where the $\rho$-functions are given by eqs. (1415).

The function $\rho_{3}(s)$ has two threshold singularities in the complex plane of total energy squared $s$. The first threshold singularity originates at the squared sum of the final-state particle masses, at

$$
s=\left(m_{1}+m_{2}+m_{3}\right)^{2},
$$


and physically it corresponds to the possibility of the system to decay into three particles (three-particle singularity in the amplitude). Another square root singularity is located in the complex plane and starts at:

$$
\begin{aligned}
s_{c u t} & =a_{c u t}-i b_{c u t}=\left(\sqrt{M_{R}^{2}-i M_{R} \Gamma_{t o t}^{R}}+m_{1}\right)^{2} \\
& \simeq\left(M_{R}+m_{1}-i \Gamma_{t o t}^{R} / 2\right)^{2} .
\end{aligned}
$$

It corresponds to the decay of the system into a particle with mass $m_{1}$ and resonance with complex mass $M_{R}-$ $i \Gamma_{\text {tot }}^{R} / 2$ (usually we assume $\Gamma_{\text {tot }}^{R}<<M_{R}$ ).
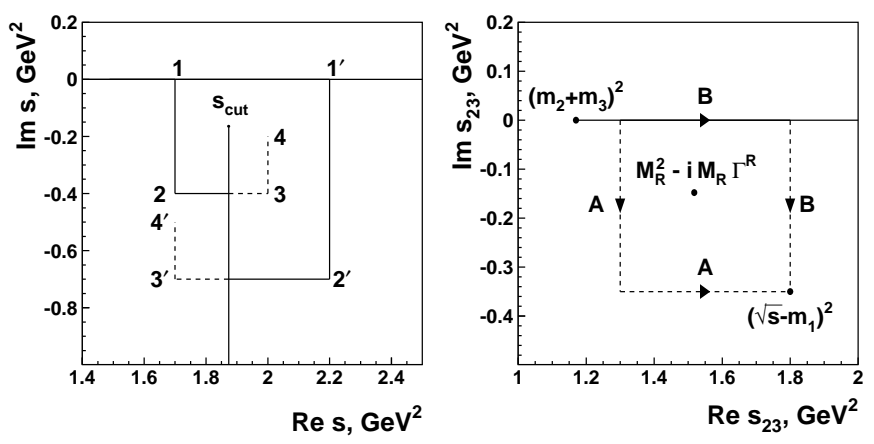

Fig. 5. The structure of threshold singularities in the $s$ and $s_{23}$ complex planes for the $\pi \Delta$ channel.

If the width of the intermediate state $(\Delta)$ tends to zero $\Gamma_{\text {tot }}^{R} \rightarrow 0$, the intermediate resonance turns into a stable particle, and the contribution from the three-particle cut disappears. For the energy region above the three-particle threshold but below the two-particle one,

$$
\left(m_{1}+m_{2}+m_{3}\right)^{2}<s<\left(M_{R}+m_{1}\right)^{2},
$$

the integrand $I\left(s, s_{23}\right)$ does not reach the singular point at $s_{23}=M_{R}^{2}$, and it is proportional to $\Gamma_{t o t}^{R}$. In the energy region above the two-particle threshold, the contribution of $I\left(s, s_{23}\right)$ is proportional to the $\delta$-function (see (94)). In the complex plane $s$, the two-body cut moves towards the real axis and finally becomes the only threshold singularity.

The influence of a pole on point of the physical region is defined by the distance between the pole position and this point. The path used for the calculation of this distance should not cross any cut. Therefore, if the pole is located on the sheet closest to the physical region, the minimal distance is defined by the imaginary part of the pole position. If the pole is located on another sheet, the minimal distance to the physical region can be estimated as a sum of distances $(i)$ between pole position and the beginning of the cut and ( $i i)$ between the beginning of the cut and real axis. Thus, if the pole is located far from the cut, only its position on the sheet closest to the physical region is important. However, if the real part of the pole position is close to $a_{c u t}$, see (127), the position on both sheets can be important to explain the behaviour of the amplitude. For example, in the decay of a resonance into
$\Delta(1232) \pi$ channel, the threshold singularity in the complex plane starts at $s \sim(1370-i 60)^{2} \mathrm{MeV}^{2}$ (see Fig. 5). This is very close to the position of the Roper resonance. Such a situation should be handled with care.

Let us formulate some rules which can be used to reach a desirable sheet in the complex s plane. The sum of integrals taken between the points $1,2,3,4$ reaches the sheet I, which is the closest one to the physical region below $a_{c u t}$ (at $\left.\operatorname{Re} s<a_{\text {cut }}\right)$ :

$$
\rho_{3}(s)=\int_{\left(m_{2}+m_{3}\right)^{2}}+\int_{\left(\sqrt{s_{1}}-m_{1}\right)^{2}}^{\left(\sqrt{s_{1}}-m_{1}\right)^{2}}+\int_{\left(\sqrt{s_{2}}-m_{1}\right)^{2}}^{\left(\sqrt{s_{2}}-m_{1}\right)^{2}}+\int_{\left(\sqrt{s_{3}}-m_{1}\right)^{2}}^{\left(\sqrt{s_{3}}-m_{1}\right)^{2}}
$$

The sum of integrals taken between points $1^{\prime}, 2^{\prime}, 3^{\prime}, 4^{\prime}$ reaches the sheet II which is the closest one to the physical region above $a_{\text {cut }}$ (at $R e s>a_{\text {cut }}$ ). In the $s_{23}$ complex plane, the different sheets are reached when the pole at $M_{R}^{2}-i M_{R} \Gamma_{t o t}^{R}$ is located on different sides of the integration path (see, for example, the paths (A) and (B) shown on the right panel of Fig. 5). The contour integral which can be constructed from such two paths is equal to the residue of the pole which is proportional to $\rho\left(s, M_{R}-\Gamma_{t o t}^{R} / 2, m_{1}\right)$. This residue provides us the difference of the $\rho_{3}(s)$ function defined on the two sheets. The sheet closest to the physical region can be reached by the integration performed first over the real axis and then by integrating over the imaginary axis in the $s_{23}$ plane. Such an integration path is close to that given by paths 1,2 and $1^{\prime}, 2^{\prime}$ in the $s$-plane and is much more convenient for practical use:

$$
\begin{aligned}
\rho_{3}(s) & =\int_{\left(m_{2}+m_{3}\right)^{2}}^{s_{23}^{i n t}} \frac{d s_{23}}{\pi} I\left(s, s_{23}\right)+\int_{s_{23}^{i n t}}^{\left(\sqrt{s}-m_{1}\right)^{2}} \frac{d s_{23}}{\pi} I\left(s, s_{23}\right) \\
s_{23}^{i n t} & =\operatorname{Re}\left(\sqrt{s}-m_{1}\right)^{2} .
\end{aligned}
$$

When $\rho_{3}(s)$ is calculated with this integral path, the function Res has, at fixed $\operatorname{Im} s$, the discontinuity at $R e s=$ $a_{\text {cut }}$ if $-\operatorname{Im} s>b_{\text {cut }}$. This is demonstrated in Fig. 6] where the real (left-hand panel) and imaginary (right-hand panel) parts of $\rho_{3}(s)$ are plotted as function of Res at fixed values of $\operatorname{Im} s_{n}$ where $n=0,30, \operatorname{Im} s_{n}=-20 M e V^{2} n$. On the physical axis $(n=0)$, the real part of $\rho_{3}(s)$ is very small up to $a_{c u t}$ and the imaginary part vanishes. At $-\operatorname{Im} s_{n}<b_{c u t}, \rho_{3}($ Res $)$ is a smooth function while at $-I m s_{n}>b_{\text {cut }}$ the function has a discontinuity at $R e s=a_{\text {cut }}$ being defined on different sheets. We plot real and imaginary parts of $\rho_{3}(R e s)$ for every $n$ with shifting the curves down by the same value $20 \mathrm{MeV}^{2}$. It is seen that when $-I m s_{n}$ reaches $b_{c u t}$, the functions have a well seen discontinuity.

\section{Conclusion}

In this paper we present further development of the spinmomentum operator expansion approach suggested ini- 

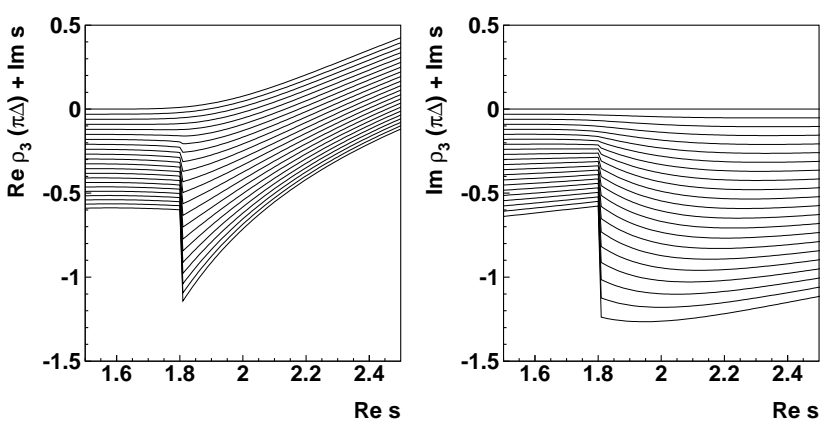

Fig. 6. The real (left-hand panel) and imaginary (right-hand panel) part of the $\rho_{3}(s)$ as a function of Res at fixed $I m s_{n}=$ $-20 n M e V^{2}, n=1,30$. For every $n$ the curves are shifted down by the same step $20 \mathrm{MeV}^{2}$.

tially in 36. Explicit expressions for cross sections and resonance partial widths are given for a large number of the pion-induced and photoproduction reactions with two or three particles in the final state. The formulae are given explicitly as they are used by the Crystal Barrel at ELSA collaboration in the analysis of single and double meson photoproduction.

\section{Acknowledgments}

We would like to thank V.V. Anisovich, L.G. Dakhno, E. Klempt and V.A. Nikonov for helpful discussions and a critical reading of the manuscript. We also thank V.A. Nikonov for the assistance with numerical calculations and figures. The work was supported by the Deutsche Forschungsgemeinschaft within the Sonderforschungsbereich SFB/TR16. We would like to thank the Alexander von Humboldt foundation for generous support in the initial phase of the project, A.V.A. for a $\mathrm{AvH}$ fellowship and A.V.S. for the Friedrich-Wilhelm Bessel award. A. Sarantsev gratefully acknowledges the support from Russian Science Support Foundation. This work is also supported by Russian Foundation for Basic Research, project no 04-0217091 and RSGSS 5788.2006.2 (Russian State Grant Scientific School).

\section{Appendix}

The operator $X_{\nu \mu_{1} \ldots \mu_{n}}^{(n+1)}$ can be written as a series of products of metric tensors and relative momentum vectors. The first term is proportional to the production of relative momentum vectors $k_{\mu}^{\perp}$, other terms correspond to the substitution of two vectors by a metric tensor with corresponding indices, and the coefficients are defined to satisfy the properties of the operator (29):

$$
\begin{array}{r}
X_{\nu \mu_{1} \ldots \mu_{n}}^{(n+1)}\left(k^{\perp}\right)=\alpha_{n+1}\left[k_{\nu}^{\perp} k_{\mu_{1}}^{\perp} k_{\mu_{2}}^{\perp} k_{\mu_{3}}^{\perp} \ldots k_{\mu_{n}}^{\perp}-\frac{k_{\perp}^{2}}{2 n+1} \times\right. \\
\left(\sum_{i=1}^{n} g_{\nu \mu_{i}}^{\perp} \prod_{j \neq i} k_{\mu_{j}}^{\perp}+\sum_{i<j}^{n} g_{\mu_{i} \mu_{j}}^{\perp} k_{\nu}^{\perp} \prod_{m \neq i \neq j} k_{\mu_{m}}^{\perp}+\ldots\right)
\end{array}
$$

$$
\begin{gathered}
+\frac{k_{\perp}^{4}}{(2 n+1)(2 n-1)}\left(\sum_{i, j<m}^{n} g_{\nu \mu_{i}}^{\perp} g_{\mu_{j} \mu_{m}}^{\perp} \prod_{l \neq i \neq j \neq m} k_{\mu_{l}}^{\perp}+\right. \\
\left.\left.\sum_{i<k, j<m}^{n} g_{\mu_{i} \mu_{k}}^{\perp} g_{\mu_{j} \mu_{m}}^{\perp} k_{\nu}^{\perp} \prod_{\substack{l \neq i \neq k \\
\neq \neq j \neq m}} k_{\mu_{l}}^{\perp}+\ldots\right)+\ldots\right] .
\end{gathered}
$$

Taking into account the tracelessness and orthogonality of the fermion propagator (50) to $\gamma^{\mu}$ one obtains:

$$
F_{\alpha_{1} \ldots \alpha_{n}}^{\mu_{1} \ldots \mu_{n}} \gamma_{\nu} X_{\nu \alpha_{1} \ldots \alpha_{n}}^{(n+1)}=F_{\alpha_{1} \ldots \alpha_{n}}^{\mu_{1} \ldots \mu_{n}} \alpha_{n+1} \hat{k}^{\perp} k_{\alpha_{1}}^{\perp} \ldots k_{\alpha_{n}}^{\perp} .
$$

Therefore, for the $\pi N$ widths of the '-' states:

$$
\begin{aligned}
& F_{\xi_{1} \ldots \xi_{n}}^{\alpha_{1} \ldots \alpha_{n}} \int \frac{d \Omega}{4 \pi} i \gamma_{\nu} \gamma_{5} X_{\nu \xi_{1} \ldots \xi_{n}}^{(n+1)}\left(k^{\perp}\right) \frac{\hat{k}_{N}+m_{N}}{2 m_{N}} i \gamma_{5} \gamma_{\eta} \times \\
& X_{\eta \beta_{1} \ldots \beta_{n}}^{(n+1)}\left(k^{\perp}\right) F_{\nu_{1} \ldots \nu_{n}}^{\beta_{1} \ldots \beta_{n}}=-F_{\xi_{1} \ldots \xi_{n}}^{\alpha_{1} \ldots \alpha_{n}} \alpha_{n+1}^{2} \frac{k_{0 N}+m_{N}}{2 m_{N}} \times \\
& \int \frac{d \Omega}{4 \pi} \hat{k}^{\perp} \hat{k}^{\perp} k_{\xi_{1}}^{\perp} \ldots k_{\xi_{n}}^{\perp} k_{\beta_{1}}^{\perp} \ldots k_{\beta_{n}}^{\perp} F_{\nu_{1} \ldots \nu_{n}}^{\beta_{1} \ldots \beta_{n}}=-F_{\xi_{1} \ldots \xi_{n}}^{\alpha_{1} \ldots \alpha_{n}} \times \\
& \frac{\alpha_{n+1}^{2}}{\alpha_{n}^{2}} \frac{k_{0 N}+m_{N}}{2 m_{N}} k_{\perp}^{2} \int \frac{d \Omega}{4 \pi} X_{\xi_{1} \ldots \xi_{n}}^{(n)}\left(k^{\perp}\right) X_{\beta_{1} \ldots \beta_{n}}^{(n)}\left(k^{\perp}\right) \times \\
& F_{\nu_{1} \ldots \nu_{n}}^{\beta_{1} \ldots \beta_{n}}=F_{\nu_{1} \ldots \nu_{n}}^{\alpha_{1} \ldots \alpha_{n}} \frac{\alpha_{n+1}}{n+1} \frac{k_{0 N}+m_{N}}{2 m_{N}}|\mathbf{k}|^{2 n+2} .
\end{aligned}
$$

Here we used property (50) as well as the fact that integral over even numbers of $k_{\mu}^{\perp}$ vectors is equal to zero.

The convolution of two gamma matrices with orbital operator is equal to zero due to tracelessness and symmetry properties:

$$
\gamma_{\mu_{1}} \gamma_{\mu_{2}} X_{\mu_{1} \ldots \mu_{n}}^{(n)}=g_{\mu_{1} \mu_{2}} X_{\mu_{1} \ldots \mu_{n}}^{(n)}+\sigma_{\mu_{1} \mu_{2}} X_{\mu_{1} \ldots \mu_{n}}^{(n)}=0
$$

Here, the second term is equal to zero being the product of the antisymmetrical and symmetrical tensors.

Other useful expressions are as follows:

$$
\begin{gathered}
F_{\mu_{1} \ldots \mu_{n}}^{\alpha_{1} \ldots \alpha_{n}} \gamma_{\nu} X_{\nu \beta \alpha_{1} \ldots \alpha_{n}}^{(n+2)}\left(k^{\perp}\right)= \\
F_{\mu_{1} \ldots \mu_{n}}^{\alpha_{1} \ldots \alpha_{n}} \alpha_{n+2}\left(\hat{k}^{\perp} k_{\beta}^{\perp} k_{\alpha_{1}}^{\perp} \ldots k_{\alpha_{n}}^{\perp}-\right. \\
\left.\frac{k_{\perp}^{2}}{2 n+3}\left(n g_{\beta \alpha_{1}}^{\perp} \hat{k}^{\perp} k_{\alpha_{2}}^{\perp} \ldots k_{\alpha_{n}}^{\perp}+\gamma_{\beta}^{\perp} k_{\alpha_{1}}^{\perp} \ldots k_{\alpha_{n}}^{\perp}\right)\right),
\end{gathered}
$$

$$
\begin{gathered}
F_{\mu_{1} \ldots \mu_{n}}^{\nu_{1} \ldots \nu_{n}} \int \frac{d \Omega}{4 \pi} X_{\beta \nu_{1} \ldots \nu_{n}}^{(n+1)}\left(k^{\perp}\right) X_{\beta^{\prime} \nu_{1}^{\prime} \ldots \nu_{n}^{\prime}}^{(n+1)}\left(k^{\perp}\right) F_{\nu_{1}^{\prime} \ldots \nu_{n}^{\prime}}^{\mu_{1}^{\prime} \ldots \mu_{n}^{\prime}} g_{\beta \beta^{\prime}} \\
=-F_{\mu_{1} \ldots \mu_{n}}^{\mu_{1}^{\prime} \ldots \mu_{n}^{\prime}}|\mathbf{k}|^{2 n+2} \frac{\alpha_{n+1}}{2 n+1}
\end{gathered}
$$

$$
\begin{gathered}
F_{\mu_{1} \ldots \mu_{n}}^{\nu_{1} \ldots \nu_{n}} \int \frac{d \Omega}{4 \pi} X_{\beta \nu_{1} \ldots \nu_{n}}^{(n+1)}\left(k^{\perp}\right) X_{\beta^{\prime} \nu_{1}^{\prime} \ldots \nu_{n}^{\prime}}^{(n+1)}\left(k^{\perp}\right) F_{\nu_{1}^{\prime} \ldots \nu_{n}^{\prime}}^{\mu_{1}^{\prime} \ldots \mu_{n}^{\prime}} k_{\beta}^{\perp} k_{\beta^{\prime}}^{\perp} \\
=F_{\mu_{1} \ldots \mu_{n}}^{\mu_{1}^{\prime} \ldots \mu_{n}^{\prime}}|\mathbf{k}|^{2 n+4} \frac{\alpha_{n}}{2 n+1},
\end{gathered}
$$

$$
F_{\alpha_{1} \ldots \alpha_{n}}^{\mu_{1} \ldots \mu_{n}} g_{\alpha_{1} \beta_{1}} O_{\beta_{2} \ldots \beta_{n}}^{\alpha_{2} \ldots \alpha_{n}} F_{\nu_{1} \ldots \nu_{n}}^{\beta_{1} \ldots \beta_{n}}=(-1)^{n} F_{\nu_{1} \ldots \nu_{n}}^{\mu_{1} \ldots \mu_{n}}
$$




\section{References}

1. C. Amsler et al. [Crystal Barrel Collaboration], Phys. Lett. B 322 (1994) 431.

2. V. V. Anisovich et al. [Crystal Ball Collaboration], Phys. Lett. B 323 (1994) 233.

3. C. Amsler et al. [Crystal Barrel Collaboration], Phys. Lett. B 333 (1994) 277.

4. C. Amsler et al. [Crystal Barrel Collaboration], Phys. Lett. B 340 (1994) 259.

5. C. Amsler et al. [Crystal Barrel Collaboration], Phys. Lett. B 355 (1995) 425.

6. C. Amsler et al., Phys. Lett. B 342 (1995) 433.

7. C. Amsler et al. [Crystal Barrel Collaboration], Phys. Lett. B 353 (1995) 571.

8. A. V. Anisovich et al., Phys. Lett. B 491 (2000) 47.

9. A. V. Anisovich et al., Phys. Lett. B 517 (2001) 261.

10. A. V. Anisovich et al., Phys. Lett. B 542 (2002) 8.

11. A. V. Anisovich et al., Phys. Lett. B 542 (2002) 19.

12. S. Godfrey and N. Isgur, Phys. Rev. D 32 (1985) 189.

13. R. Ricken, M. Koll, D. Merten, B. C. Metsch and H. R. Petry, Eur. Phys. J. A 9, 221 (2000) arXiv:hep-ph/0008221.

14. N. Isgur, 7th International Conference on the Structure of Baryons, (B.F. Gibson et al. eds.), Santa Fe, New Mexico, 3-7 Oct 1995, World Scientific, Singapore, 1996.

15. S. Capstick and N. Isgur, Phys. Rev. D 34 (1986) 2809. S. Capstick and W. Roberts, Prog. Part. Nucl. Phys. 45 (2000) S241.

16. U. Loring, B. C. Metsch and H. R. Petry, Eur. Phys. J. A 10 (2001) 447 arXiv:hep-ph/0103290.

17. D. Diakonov, V. Petrov and M. V. Polyakov, Z. Phys. A 359 (1997) 305 arXiv:hep-ph/9703373.

18. P. Bicudo and G. M. Marques, Phys. Rev. D 69 (2004) 011503 arXiv:hep-ph/0308073.

19. D. Jido, J. A. Oller, E. Oset, A. Ramos and U. G. Meissner, Nucl. Phys. A 725 (2003) 181 arXiv:nucl-th/0303062.

20. V. V. Anisovich, S.M. Gerasyuta and A. V. Sarantsev, Int. J. Mod. Phys. A 6 (1991) 625.

21. E. Klempt, Phys. Rev. C $66 \quad$ (2002) 058201 arXiv:hep-ex/0206012.

22. V. V. Anisovich, M.N. Kobrinsky, J.Nyiri, Yu.M. Shabelski. "Quark model and high energy collisions", second edition, World Scientific, Singapure, 2004.

23. A. V. Anisovich, V. V. Anisovich and A. V. Sarantsev, Phys. Rev. D 62 (2000) 051502 arXiv:hep-ph/0003113.

24. O. Bartholomy et al., Phys. Rev. Lett. 94 (2005) 012003.

25. V. Crede et al., Phys. Rev. Lett. 94 (2005) 012004.

26. B. Krusche et al., Phys. Rev. Lett. 74 (1995) 3736.

27. R. Beck et al., Phys. Rev. Lett. 78 (1997) 606.

28. O. Bartalini et al. [Graal collaboration], submitted Eur. Phys. J. A.

29. D. Rebreyend et al.,Nucl. Phys. A 663 (2000) 436.

30. J. Ajaka et al., Phys. Rev. Lett. 81 (1998) 1797.

31. K. H. Glander et al., Eur. Phys. J. A 19 (2004) 251.

32. R. Lawall et al., Eur. Phys. J. A 24 (2005) 275.

33. J. W. C. McNabb et al., Phys. Rev. C 69 (2004) 042201.

34. B. Carnahan, "Strangeness photoproduction in the $\gamma \mathrm{p} \rightarrow$ $\mathrm{K}^{0} \Sigma^{+}$reaction", UMI-31-09682 (microfiche), Ph.D. thesis (2003) at the Catholic University of America, Washington, D.C.

35. R. G. T. Zegers et al., Phys. Rev. Lett. 91 (2003) 092001.
36. A. Anisovich, E. Klempt, A. Sarantsev and U. Thoma, Eur. Phys. J. A 24 (2005) 111.

37. A. V. Anisovich, A. Sarantsev, O. Bartholomy, E. Klempt, V. A. Nikonov and U. Thoma, Eur. Phys. J. A 25, 427 (2005) arXiv:hep-ex/0506010.

38. A. V. Sarantsev, V. A. Nikonov, A. V. Anisovich, E. Klempt and U. Thoma, Eur. Phys. J. A 25, 441 (2005) arXiv:hep-ex/0506011.

39. A. V. Anisovich, V. V. Anisovich, V. N. Markov, M. A. Matveev and A. V. Sarantsev, J. Phys. G 28 (2002) 15 arXiv:hep-ph/0105330. 